\title{
The cost-effectiveness of antenatal and postnatal education and support interventions for women aimed at promoting breastfeeding in the UK
}

Ifigeneia Mavranezouli, 2,2, Jo Varley-Campbell ${ }^{1,2}$, Sarah Stockton², Jennifer Francis ${ }^{2}$, Clare Macdonald ${ }^{3,4}$, Sunita Sharma ${ }^{5}$, Peter Fleming ${ }^{6}$, Elizabeth Punter ${ }^{7}$, Charlotte Barry ${ }^{8}$, Maija Kallioinen², Nina Khazaezadeh ${ }^{9}$ and David Jewell ${ }^{10}$

\begin{abstract}
Background: Breastfeeding is associated with health benefits to mothers and babies and cost-savings to the health service. Breastfeeding rates in the UK are low for various reasons including cultural barriers, inadequate support to initiate and sustain breastfeeding, lack of information, or choice not to breastfeed. Education and support interventions have been developed aiming at promoting breastfeeding rates. The objective of this study was to assess the cost-effectiveness of such interventions for women, initiated antenatally or in the first 8 weeks postnatally, aiming at improving breastfeeding rates, in the UK.
\end{abstract}

Methods: A decision-analytic model was constructed to compare costs and quality-adjusted life-years (QALYS) of a breastfeeding intervention from the perspective of health and personal social services in England. Data on intervention effectiveness and the benefits of breastfeeding were derived from systematic reviews. Other model input parameters were obtained from published sources, supplemented by expert opinion.

Results: The incremental cost-effectiveness ratio (ICER) of the modelled intervention added on standard care versus standard care was $£ 51,946 /$ QALY, suggesting that the intervention is not cost-effective under National Institute for Health and Care Excellence (NICE) criteria in England. Sensitivity analysis suggested that the cost-effectiveness of the intervention improved as its effectiveness increased and intervention cost decreased. At the base-case effect (increase in breastfeeding rates 16-26 weeks after birth by $19 \%)$, the intervention was cost-effective $(<£ 20,000 /$ QALY) if its cost per woman receiving the intervention became $\approx £ 40-£ 45$. At the base-case cost $(£ 84)$, the intervention was costeffective if it increased breastfeeding rates by at least $35-40 \%$.

Conclusions: Available breastfeeding interventions do not appear to be cost-effective under NICE criteria in England. Future breastfeeding interventions need to have higher effectiveness or lower cost compared with currently available interventions in order to become cost-effective. Public health and other societal interventions that protect, promote and support breastfeeding may be key in improving breastfeeding rates in the UK.

\footnotetext{
*Correspondence: i.mavranezouli@ucl.ac.uk

${ }^{1}$ Centre for Outcomes Research and Effectiveness, Research Department

of Clinical, Educational \& Health Psychology, University College London,

1-19 Torrington Place, London WC1E 7HB, UK

Full list of author information is available at the end of the article
}

(c) The Author(s) 2022. Open Access This article is licensed under a Creative Commons Attribution 4.0 International License, which permits use, sharing, adaptation, distribution and reproduction in any medium or format, as long as you give appropriate credit to the original author(s) and the source, provide a link to the Creative Commons licence, and indicate if changes were made. The images or other third party material in this article are included in the article's Creative Commons licence, unless indicated otherwise in a credit line to the material. If material is not included in the article's Creative Commons licence and your intended use is not permitted by statutory regulation or exceeds the permitted use, you will need to obtain permission directly from the copyright holder. To view a copy of this licence, visit http://creativecommons.org/licenses/by/4.0/. The Creative Commons Public Domain Dedication waiver (http://creativeco mmons.org/publicdomain/zero/1.0/) applies to the data made available in this article, unless otherwise stated in a credit line to the data. 
Keywords: Breastfeeding interventions, Decision-analytic modelling, Economic evaluation, Cost-effectiveness

\section{Background}

Breastfeeding is associated with important health benefits to both babies and breastfeeding women [1]. Nevertheless, global rates of breastfeeding are well below targets set by the World Health Assembly [2]. The UK has some of the lowest breastfeeding rates in the world with reported barriers to breastfeeding including a lack of access to support services in the community and at work, cultural barriers and misinformation on the benefits and practicalities of infant feeding [3]. Various interventions have been developed over the years, aimed at promoting initiation and/or maintenance of breastfeeding [4-6]. Existing evidence on the cost-effectiveness of such interventions is limited and has not considered the long-term benefits to women and their babies and related costsavings associated with breastfeeding [7-11]. The objective of this study was therefore to examine the long-term cost-effectiveness of interventions for women, initiated antenatally or in the first 8 weeks after birth, aimed at promoting initiation and/or maintenance of breastfeeding from the perspective of the National Health Service (NHS) and Personal Social Services (PSS) in England, using decision-analytic economic modelling.

The analysis presented here is part of the work that informed the updating of national guidance on postnatal care in England, published by the National Institute for Health and Care Excellence (NICE) [12]. The guideline was developed by a guideline committee, an independent multi-disciplinary group of clinical academics, health professionals and service users and carers with expertise and experience in the area of postnatal care. The committee contributed to the development of the economic model by providing advice on issues relating to the provision of breastfeeding interventions and associated benefits and resource use savings. They also contributed to the economic model structure and advised on model inputs in areas where there was paucity of evidence.

\section{Methods}

\section{Population}

The study population comprised women who are pregnant or have given birth to healthy babies at term, and their babies. The women's age at the start of the economic model was 30 years, as this is the mean age of women giving birth in England and Wales [13]. The starting age of the cohort was needed in order to model lifetime benefits to women associated with breastfeeding. In sensitivity analyses we varied the age of women when receiving the intervention from 25 to 35 years to explore whether this has an impact on the results. Women could have had a singleton, twin or higher order live birth. In accordance with national epidemiological data, the mean number of babies per delivery of liveborns was 1.016 [14].

Women received the intervention at one point in time, and, in the base-case analysis, the intervention's effect was applied only on that birth and not on future ones. In sensitivity analysis we maximised the impact of the intervention by assuming that all women received the intervention when they had their first baby and that the effect of the intervention on breastfeeding rates would be retained in all subsequent births, using the total fertility rate of 1.70 births/woman in England [14].

\section{Intervention}

The characteristics of the breastfeeding intervention regarding effectiveness and resource use (number of sessions, format, people delivering the intervention, etc.) were informed by the findings of a systematic review and meta-regression of 62 randomised controlled trials (RCTs) undertaken to inform the NICE guideline [15]; resource use characteristics were further supplemented by the committee's expert opinion to reflect routine practice in the UK. The focus of the analysis was an intervention for women that comprised education, advice or support from a peer or professional, provided postnatally and initiated antenatally or within the first eight weeks after birth, as the majority of clinical evidence was available for this type of intervention. Broader public health interventions that aim to promote breastfeeding were beyond the guideline scope. In accordance with available evidence, the intervention was assumed to be provided in addition to standard care; the comparator of the analysis was standard care alone. The definition of standard care varied widely across RCTs included in the systematic review and meta-regression that informed the economic analysis. Standard care ranged from no intervention, through written materials and peer breastfeeding support, to availability of breastfeeding educational programmes of variable intensity in-hospital or in the community. In England, standard care is also variable and may include provision of written material, antenatal breastfeeding educational programmes, and postnatal breastfeeding support groups run by peers and/or health professionals; in most settings breastfeeding information and support is provided by midwives and health visitors as part of routine postnatal care visits.

In order to specify the intervention and identify its effective components, effectiveness data on 'any 
Table 1 Effectiveness of interventions aimed at promoting breastfeeding - results of meta-regression for 'any breastfeeding 16 to 26 weeks after birth' [15]

\begin{tabular}{|c|c|c|c|}
\hline Comparisons - every component vs standard care & Risk Ratio & Lower $95 \% \mathrm{Cl}$ & Upper $95 \% \mathrm{Cl}$ \\
\hline Any intervention & 1.08 & 1.03 & 1.13 \\
\hline \multicolumn{4}{|l|}{ How } \\
\hline Face-to-face individual & 1.07 & 1.01 & 1.14 \\
\hline Face-to-face group & 1.95 & 1.45 & 2.27 \\
\hline Remote & 1.15 & 1.05 & 1.26 \\
\hline Self-help & 1.06 & 0.74 & 1.40 \\
\hline \multicolumn{4}{|l|}{ Number of Contacts } \\
\hline 0 & 1.18 & 0.96 & 1.39 \\
\hline 1 & 1.05 & 0.95 & 1.14 \\
\hline $2-3$ & 1.07 & 0.97 & 1.17 \\
\hline 4-8 & 1.19 & 1.10 & 1.30 \\
\hline 9 & 1.13 & 1.00 & 1.26 \\
\hline \multicolumn{4}{|l|}{ Duration of intervention } \\
\hline Less than 8 weeks & 1.04 & 0.97 & 1.10 \\
\hline More than 8 weeks & 1.20 & 1.11 & 1.29 \\
\hline \multicolumn{4}{|l|}{ Where delivered } \\
\hline Home & 1.12 & 1.05 & 1.19 \\
\hline Healthcare setting & 1.06 & 0.96 & 1.17 \\
\hline Mixed home/healthcare setting & 1.16 & 1.03 & 1.30 \\
\hline
\end{tabular}

Comparisons with statistically significant effects at the $p \leq 0.05$ level have been highlighted in bold. Cl confidence interval

breastfeeding between 16 and 26 weeks after birth', obtained from the systematic review and meta-regression, were inspected (Table 1). Data on 'any breastfeeding', rather than 'exclusive breastfeeding' were selected because most of the available data on the protective effects of breastfeeding that informed the economic model were relevant to 'any' breastfeeding (more versus less, longer versus shorter duration, any versus none, etc.) rather than 'exclusive' breastfeeding; moreover, the period of 16-26 weeks after birth was chosen to ensure that breastfeeding was established and therefore could have an impact on longer-term mother and baby outcomes, and during this period no efficacy data on exclusive breastfeeding were available. The components of the modelled intervention were specified by looking at the intervention characteristics that demonstrated a statistically significant effect (risk ratio, RR) versus standard care. Face-to-face interventions, delivered either individually or in group format, and also interventions delivered remotely, appeared to be effective compared with standard care. However, the group intervention effect was by far the largest observed in our meta-regression and only based on a single small study ( $N=100$; in comparison, the total number of participants across studies included in that meta-regression was $N=14,229$ ); hence the committee did not consider the group intervention effect further when specifying the characteristics of the modelled intervention. Interventions comprising $4-8$ contacts appeared to have the greatest effect. Interventions seemed to be effective if they were delivered at home or in a mixed home and healthcare setting.

\section{Effectiveness of the modelled intervention}

The economic analysis utilised the effect on any breastfeeding at 16-26 weeks after birth for "4-8 contacts vs standard care" [mean RR 1.19, 95\% confidence intervals (CI) 1.10 to 1.30 ] [15]. Sensitivity analysis explored the impact of changes in the mean effect (range of RR from 1.05 to 2.00) on the cost-effectiveness of the intervention.

\section{Modelled intervention cost}

The intervention cost was estimated using national unit costs [16] and expert advice, assuming that the intervention consisted of 6 contacts, i.e. the average of 4-8 contacts which was the most effective number of contacts identified in the meta-regression [15]. Based on the committee's advice on patterns of routine practice in England, we made the following assumptions regarding the delivery of the intervention: four contacts comprised 30-min individual face-to-face sessions, and two further contacts comprised 45-min group face-to-face sessions delivered to groups of 6 women. The first two individual sessions were provided by a health professional on a NHS England Agenda for Change Band 5 salary. The remaining two 
Table 2 Cost of intervention aimed at promoting breastfeeding

\begin{tabular}{|c|c|c|}
\hline Cost element & Unit cost & $\begin{array}{l}\text { Cost per } \\
\text { woman }\end{array}$ \\
\hline $\begin{array}{l}2 \text { individual face-to-face sessions lasting } 30 \text { min each (total } 60 \text { min), provided by a health professional in NHS } \\
\text { England Agenda for Change (AfC) Band } 5 \text { (nursing, midwifery and health visiting staff). }\end{array}$ & $£ 59$ per patient-related hour a & $£ 59$ \\
\hline $\begin{array}{l}2 \text { individual face-to-face sessions lasting } 30 \mathrm{~min} \text { each (total } 60 \mathrm{~min} \text { ), delivered by a volunteer trained peer } \\
\text { supporter. }\end{array}$ & $£ 20$ per patient-related hour ${ }^{b}$ & $£ 20$ \\
\hline $\begin{array}{l}2 \text { group face-to-face sessions delivered to groups of } 6 \text { women, lasting } 45 \text { min each (total } 90 \text { min } / 6 \\
\text { women }=15 \text { min per woman), delivered by a volunteer trained peer supporter. }\end{array}$ & £20 per patient-related hour ${ }^{b}$ & $£ 5$ \\
\hline TOTAL COST PER WOMAN & & $£ 84$ \\
\hline
\end{tabular}

individual and the two group sessions were provided by a volunteer trained peer supporter. The total estimated intervention cost was $£ 84$ (Table 2 ). The intervention was offered in addition to standard care, and therefore the description and cost of standard care was omitted from both arms of the model. Sensitivity analysis explored the impact of changes in the intervention cost (range from $£ 20$ to $£ 100$ ) on the cost-effectiveness of the intervention.

\section{Baseline probability of breastfeeding}

Current breastfeeding rates under standard care for the period of 16 weeks ( 4 months) to 26 weeks ( 6 months) after birth were obtained from national statistics.

For baby outcomes, baseline rates of any breastfeeding at 4 months after birth were used, as breastfeeding is established by this time point, leading to health benefits, and evidence suggests that the protective effect of breastfeeding is retained after this point even after breastfeeding stops [1]. For breast cancer in mothers, baseline rates of any breastfeeding at 6 months after birth were used, as evidence suggests that the effect of breastfeeding on the incidence of breast cancer may be significant from 6 months of breastfeeding onwards $[17,18]$.

The most recent (2019) data on any breastfeeding were only available for 6-8weeks after birth [19]. The most recent rates of any breastfeeding at 4 and 6 months after birth in England were available for the year 2010 [20]. Nevertheless, it was possible to estimate the rates of any breastfeeding at 4 and 6 months after birth for 2019, using the 2019 data on the prevalence of any breastfeeding at 6-8 weeks and the instant rate of reduction in any breastfeeding between 6 weeks and 4 months (16 weeks) and between 4 months and 6 months ( 26 weeks) as calculated from the available 2010 data. In order to estimate the rates of breastfeeding at 4 and 6 months after birth in 2019 using the available data, we assumed an exponential decrease in breastfeeding rates, which was more
Table 3 Prevalence of any breastfeeding in England at different time points after birth

\begin{tabular}{|c|c|c|}
\hline \multirow[t]{2}{*}{ Time point } & \multicolumn{2}{|c|}{ Prevalence of any breastfeeding } \\
\hline & $2010[20]$ & 2019 [19] \\
\hline Birth & $83 \%$ & \\
\hline 6-8 weeks after birth & $57 \%$ [6 weeks] & $\begin{array}{l}53 \% \text { [6-8 weeks, cases } \\
\text { with known status } \\
\text { only] }\end{array}$ \\
\hline 4 months after birth & $44 \%$ & $42 \%$ [estimated] $^{\mathrm{a}}$ \\
\hline 6 months after birth & $36 \%$ & $34 \%$ [estimated] ${ }^{a}$ \\
\hline
\end{tabular}

a estimated using the 2019 figure for the prevalence of any breastfeeding at 6-8 weeks and the instant rate of reduction in any breastfeeding between 6 weeks and 4 months, and between 4 months and 6 months, as calculated from the 2010 data (assuming exponential decrease in breastfeeding rates)

rapid between 6 weeks and 4 months compared with the period between 4 and 6 months, according to available data. This assumption was necessary due to lack of more detailed data that would allow us to determine the rate of decrease in breastfeeding rates more accurately over time. The actual and estimated rates of any breastfeeding at different time points following birth for the years 2010 and 2019 are shown in Table 3.

\section{Overview of costs and benefits considered in the analysis}

Costs consisted of the intervention cost and costs associated with breastfeeding outcomes that are incurred in community, primary or secondary healthcare or personal social service settings. Costs to parents relating to either formula feeding (milk powder, bottles, sterilising equipment) or breastfeeding (breast pumps, bottles, sterilising equipment, nursing bras, nipple pads) were not considered as these were outside the NHS/PSS perspective of the analysis. The cost year was 2018. The primary outcome measure was the QALY. Other secondary outcome measures were determined by, and are specific to, the clinical conditions considered in the analysis, 
Table 4 Clinical conditions associated with breastfeeding that were considered in the economic analysis

Clinical conditions in babies
- Gastrointestinal infection (GI) [diarrhoea attributable to infection]
- Respiratory tract infection (RTI)
- Acute otitis media (AOM)
- Mortality due to infectious diseases
- Mortality due to sudden infant death syndrome (SIDS)

Clinical conditions in mothers

- Breast cancer

and are described under 'Model structure' together with each relevant model component.

\section{Selection of clinical conditions associated with breastfeeding for inclusion in modelling}

A systematic review of studies that modelled long-term clinical benefits to mothers and babies (and/or related cost-savings to health and personal social services) associated with breastfeeding was undertaken in order to identify data on long-term clinical outcomes associated with breastfeeding, as well as relevant epidemiological and resource use data that could be adopted or adapted to inform our economic analysis. Details of the review are provided in the guideline evidence report [15]. Included studies and studies excluded after full text was obtained are provided in Supplementary File 1.

The review identified two studies of high quality and directly relevant to our study's objective, that is, the modelling of long-term outcomes and cost-savings associated with breastfeeding [1, 18]. Renfrew et al. [18] developed an economic model to estimate long-term benefits to mothers and babies and cost-savings to the UK healthcare system associated with breastfeeding. The study, which was commissioned by UNICEF UK, was informed by high quality systematic reviews regarding the benefits of breastfeeding to mothers and babies. Victora et al. [1] examined the association between breastfeeding and clinical outcomes to mothers and babies based on the results of 28 systematic reviews and meta-analyses, 22 of which were commissioned by the World Health Organization (WHO). These two studies alone reported all data on the association between breastfeeding and mother and baby outcomes that had informed the remaining modelling studies. Only one modelling study [21] used data on the association between breastfeeding and breast cancer that had not been already reported by either Renfrew et al. [18] or Victora et al. [1], which were obtained from a more recent meta-analysis published in 2017 [22].

Based on the review findings, we decided to use the analysis undertaken by Renfrew et al. [18] as the starting point for our analysis, regarding the selection and modelling of clinical benefits (and related NHS/PSS cost-savings) associated with breastfeeding, and update the data on the association between breastfeeding and clinical outcomes using, where available, more up-to-date evidence reported in Victora et al. [1]. The Renfrew et al. study [18] was selected for this purpose because it considered a range of clinical conditions, was of high quality and utilised UK-specific epidemiological and resource use data. Based on the evidence from these two studies, we selected clinical conditions associated with breastfeeding for inclusion in our analysis, also taking into account feasibility issues and the expected magnitude of clinical benefits and cost-savings per person associated with a change in breastfeeding rates. The evidence we reviewed and the considerations that led to selection of clinical conditions are shown in Supplementary File 2. The clinical conditions associated with breastfeeding that were selected for inclusion in our economic analysis are shown in Table 4.

\section{Model structure}

A hybrid decision-analytic model was constructed using Microsoft Office Excel 2013 to estimate total NHS/PSS costs and benefits to mothers and babies associated with the provision of a breastfeeding intervention. The structure of the model, which aimed to simulate the course of a number of clinical conditions whose incidence is associated with breastfeeding, was driven by patterns of clinical practice in the UK and the availability of relevant clinical data.

According to the model structure, hypothetical cohorts of women who are pregnant or have given birth to healthy babies at term were either initiated on a breastfeeding intervention in addition to standard care, or received standard care only. Following care, women either breastfed or did not breastfeed their babies at 16-26 weeks after birth. Women and their babies were subsequently followed up for a period of time that ranged from one year after birth to lifetime, depending on the clinical condition assessed, to estimate their outcomes and associated costs resulting from their breastfeeding status at 16-26 weeks after birth.

The first part of the economic model, which assessed the impact of the breastfeeding intervention on breastfeeding rates at 16-26weeks after birth (effectiveness of intervention), took the form of a decision tree. This was followed by separate models on each of the clinical 
conditions considered for mothers and babies, which took the form of either decision trees or Markov models, as appropriate for the condition examined.

The models on gastrointestinal infection (GI), respiratory tract infection (RTI) and acute otitis media (AOM) in babies took the form of a simple decision tree, where babies either developed one of the infections or not, following the structure in the Renfrew et al. model [18]. Those who developed an infection were treated by GPs (with each infection assumed to correspond to one GP contact), with a number of babies developing GI and RTI being hospitalised for further treatment. The time horizon of those models was one year. The outcome measures were the number of cases of GI, lower RTI and AOM as well as the number of hospitalisations due to GI and RTI in babies aged up to one year that were prevented by breastfeeding. These were secondary outcomes in the analysis.

One model was developed for mortality due to infectious diseases or sudden infant death syndrome (SIDS) in babies. Babies who did not die due to infectious diseases or SIDS over their first year of life owing to the protective effect of breastfeeding entered a very simple, two-state Markov model, with a one-year cycle, that considered the states of 'alive' and 'dead' over the babies' lifetimes. This was a new model as Renfrew et al. [18] did not consider mortality due to infectious diseases in their economic modelling, and assessed cost-savings and outcomes associated with SIDS in a narrative synthesis. The outcome measures of this model component were the number of QALYs gained over saved babies' lifetime (primary outcome) and the number of deaths due to infectious diseases or SIDS prevented in babies aged up to one year (secondary outcome).

One three-state Markov model was developed to assess costs and outcomes for women at risk for breast cancer starting from 30 years of age and over their lifetime. The model, which considered the protective effect of breastfeeding on the risk of breast cancer over women's lifetime, included the states of 'no breast cancer', 'breast cancer' and 'death'; the model cycle was one year and a half-cycle correction was applied. This model had the same overall structure as the Renfrew et al. study [18], but adopted a different approach and considered more parameters associated with the risk of breast cancer in parous women, employed different assumptions to model the course of disease (in particular mortality), and utilised different epidemiological, utility and cost data on breast cancer.

Breast cancer in women who survived was assumed to last 10 years, after which women who survived re-entered the 'no breast cancer' state and were at risk of developing a new breast cancer. The state of 'breast cancer' consisted of 10 tunnel states, one for each year of breast cancer, so that the time women spent with breast cancer could be estimated and a breast cancer's duration-dependent mortality, as well as time-dependent costs and utilities associated with breast cancer, could be applied. The outcome measures of this model component were the number of QALYs (primary outcome) and the number of new cases of breast cancer prevented over lifetime (secondary outcome).

The overall structure of the economic model assessing the cost-effectiveness of an intervention for starting and maintaining breastfeeding is shown in Fig. 1. Figure 2 shows the economic model component on mothers' breast cancer.

Our model was built on the evidence that provision of a breastfeeding intervention increases breastfeeding rates; the protective effect of increased breastfeeding on various clinical conditions was subsequently applied onto the current (baseline) incidence of each clinical condition under standard care, to estimate the reduction in incidence following intervention. The model took into account the fact that the current (baseline) incidence of each clinical condition reflects the current mix of mothers and babies who breastfeed/are breastfed and those who do not breastfeed/are not breastfed, respectively (i.e. all mothers and their healthy babies born at term under standard care).

To estimate the incidence of each clinical condition under current standard practice in mothers that breastfeed or babies that are breastfed (BF) and those that do not breastfeed / are not breastfed, respectively (nonBF), the following formulae were used [23]:

$$
\text { Incidence in nonBF }=\frac{\text { Overall incidence }}{\text { Current BF rate } x R R+1-\text { current BF rate }}
$$

and

\section{Incidence in $B F=$ Incidence in non $B F \times R R$}

where 'overall incidence' is the incidence of the clinical condition in the overall population of mothers or babies, and $R R$ the risk ratio expressing the protective effect of breastfeeding on the clinical condition assessed. These formulae utilise $R R$ to express the protective effect of breastfeeding. On the other hand, the protective effect of breastfeeding has been expressed as odds ratio (OR) for some clinical conditions. However, when the incidence of an event at baseline is rare $(<10 \%)$, then OR approximates $R R$ and the formulae can produce accurate results using OR instead of RR [24]. 


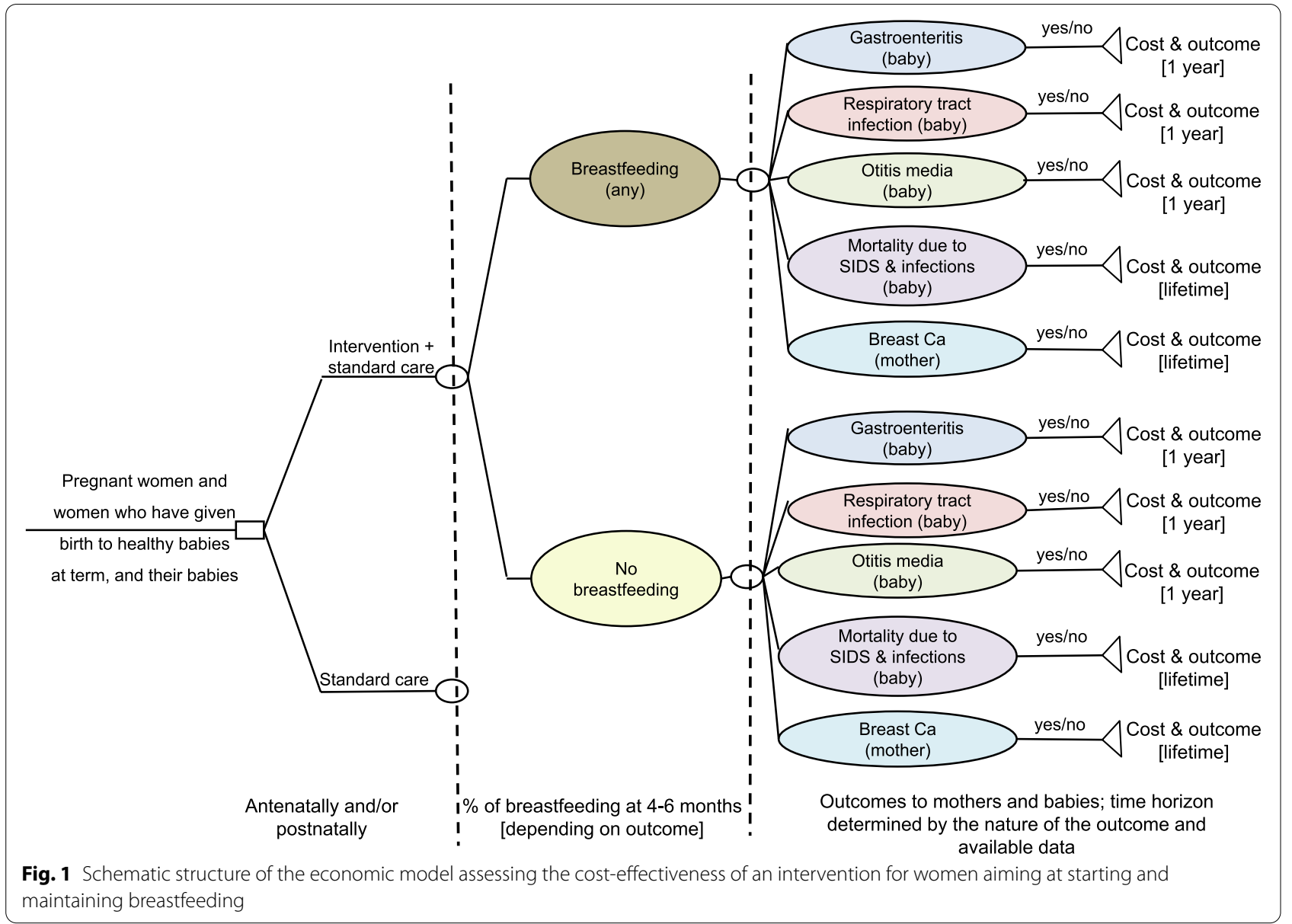

\section{Data on the association of breastfeeding with the clinical conditions considered in the economic analysis}

We utilised data from Victora et al. [1] for the following outcomes:

i) incidence of diarrhoea (reflecting GI) in babies and children aged 6 months - 5 years (RR of more versus less breastfeeding: $0.46,95 \%$ CI 0.28 to 0.78 )

ii) hospitalisation due to diarrhoea (reflecting GI) in babies and children aged $\leq 5$ years (RR of more versus less breastfeeding: $0.28,95 \%$ CI 0.16 to 0.50 )

iii) incidence or prevalence of lower RTI in babies and children aged <2years (RR of more versus less breastfeeding: $0.68,95 \%$ CI 0.60 to 0.77 )

iv) hospitalisation due to RTI (both lower and upper, according to available evidence) in babies and children aged $<2$ years ( $R R$ of more versus less breastfeeding: $0.43,95 \%$ CI 0.33 to 0.55 )

v) incidence of $\mathrm{AOM}$ in babies and children aged $<2$ years (OR of more versus less breastfeeding: 0.67 , $95 \%$ CI 0.62 to 0.72 ) vi) mortality due to infectious diseases in babies and children aged 6-23 months (OR of any versus no breastfeeding: $0.48,95 \%$ CI 0.38 to 0.60 )

vii) mortality due to SIDS in babies and children aged $\geq 2$ months (OR of any versus no breastfeeding: 0.38 , $95 \%$ CI 0.27 to 0.54 ).

Updated evidence suggests a protective effect of breastfeeding on the incidence of and hospitalisations due to GI in babies over the first 5 years of their life, although this effect appears to be stronger in younger ages. Moreover, breastfeeding is associated with a reduction in the incidence of lower RTI and AOM and hospitalisation due to RTI in babies over the first 2 years of their life. Nevertheless, we conservatively estimated costs and outcomes associated with incidence of GI, lower RTI and AOM and hospitalisation due to GI and RTI in babies up to their first year of life, to retain consistency with the Renfrew et al. model [18] and because relevant epidemiological data were available for babies up to one year of age. 


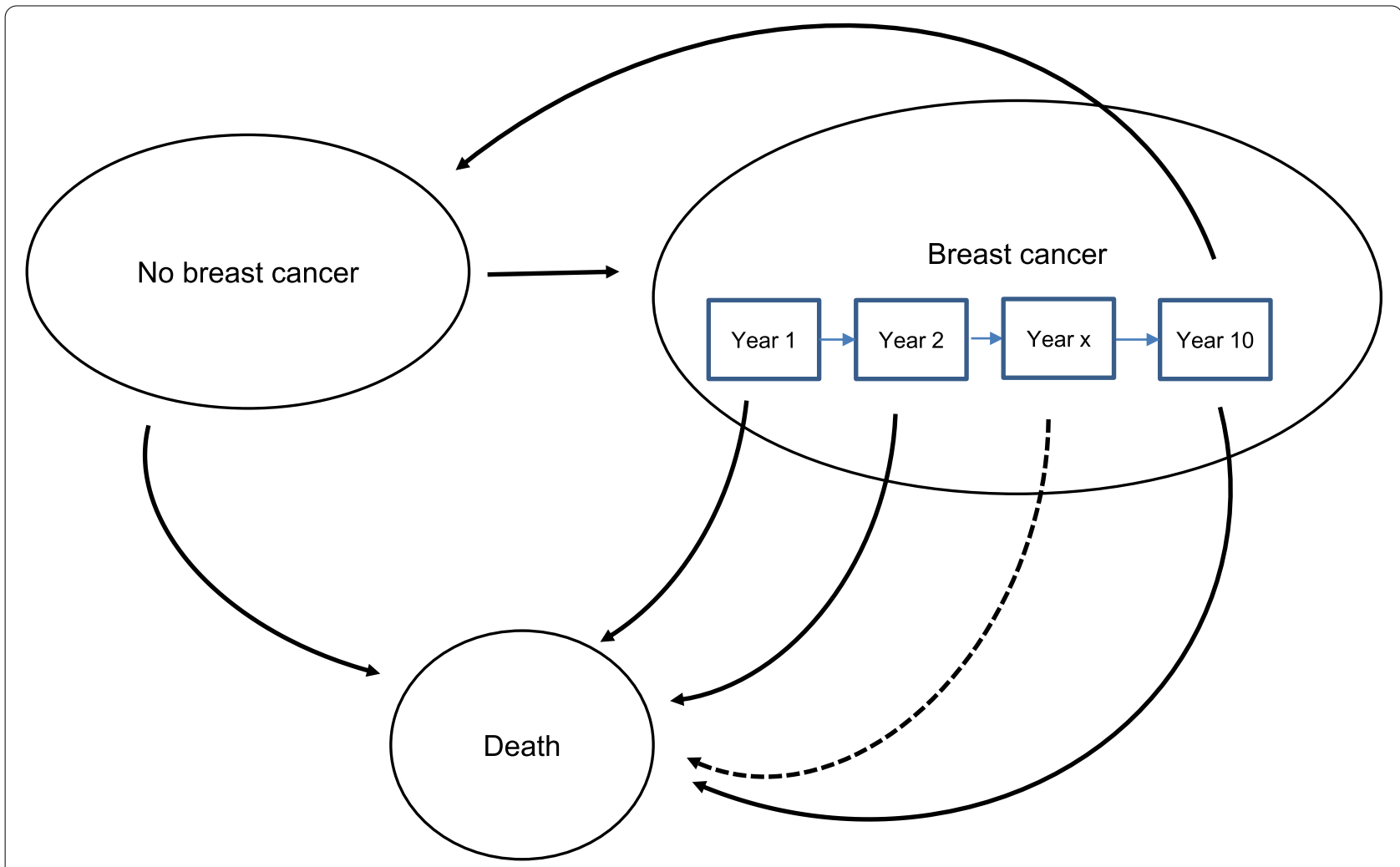

Fig. 2 Schematic structure of the economic model component on mothers' breast cancer

Data on the protective effect of breastfeeding on the incidence of breast cancer were obtained from a published meta-analysis [22], which pooled data from 25 studies on parous women and adjusted for several confounders such as age, parity, age at first pregnancy and family history of breast cancer. The standardised RR for breast cancer in parous women for any versus no breastfeeding for 6 months was used $(0.86,95 \%$ CI 0.82 to 0.91$)$.

\section{Baseline risks and mortality}

For the baseline incidence of GI, lower RTI and AOM in babies aged 0-1 years in England we used the number of GP consultations for this age group for the clinical diagnoses of (a) diarrhoea, intestinal infectious diseases, noninfective enteritis, and colitis $(4682$ per 100,000$)$ to reflect consultations for GI; (b) lower RTI (23,433 per 100,000); and (c) AOM (13,556 per 100,000). These data were reported in Renfrew et al. [18], derived from the Royal College of General Practitioners (RCGP) database.

The baseline rates of hospital admissions due to GI and RTI over the first year of life (15.3 and 115.4 per 1000 live births, respectively) were estimated using data on admissions for infectious intestinal diseases (reflecting GI) and RTI in babies aged 0-1 years in England [25], divided by the population aged 0-1 years in England [26].
The baseline mortality due to infectious diseases (12 per 100,000) and SIDS (25 per 100,000) in babies aged $0-1$ years was estimated by dividing the number of deaths due to infectious diseases and SIDS in babies aged $0-1$ years with the number of live births, using infant mortality data in England and Wales [27].

To estimate the annual mortality of babies who did not die from infectious diseases or SIDS owing to the protective effect of breastfeeding we used the proportion of males/females aged one year in England [26] and ageand gender-specific mortality over lifetime [28].

The baseline incidence of breast cancer in parous women was estimated using:

- The age-specific incidence of breast cancer in women in the general population, i.e. a mixture of parous and nulliparous women [29] (see Supplementary File 3).

- The percentage of nulliparous women in the population of women aged 30 years and over. This was $48 \%$ at 30 years of age; $27 \%$ at 35 years of age; $19 \%$ at 40 years of age; and $18 \%$ at 45 years of age and above [30].

- The mean number of children per parous woman aged $\geq 30$ years (including previous births), which was approximately 2 , starting from 1.90 at 30 years 
of age and reaching 2.23 at 45 years of age [30]. This parameter was used to estimate the incidence of breast cancer in parous women, as parity reduces the incidence of breast cancer and the reduction depends on the number of children per woman.

- The protective effect of parity on breast cancer, expressed as an OR of incidence of breast cancer in parous women with 2 live births versus non-parous women $(0.84,95 \%$ CI 0.80 to 0.89) [31]. Parous women with 2 live births were selected as the relevant sub-population of parous women, as the mean number of children of parous women aged $\geq 30$ years (which is the study population) is 2 , as reported above.

For every year in the model, the incidence of breast cancer in parous and nulliparous women was estimated using the formulae described earlier [23], using the overall age-specific incidence of breast cancer in women in the general population, the percentage of nulliparous women amongst women in the general population, and the protective effect of parity on breast cancer. The same formulae were used to estimate the incidence of breast cancer under current standard practice in women aged $\geq 30$ years who breastfed and those who did not, amongst parous women.

Mortality in women without breast cancer was derived from age-specific mortality data for women in the general population [28]. For women with breast cancer, mortality in every model cycle was estimated using age-specific data on mortality of women in the general population [28], age-specific data on mortality due to breast cancer in women in the general population [32] (see Supplementary File 3), and age-adjusted net survival data for women with breast cancer over 1-10 years after diagnosis [33]. Using these data and a number of assumptions, it was possible to estimate the age- and breast cancer's duration-specific mortality in women with breast cancer, depending on the number of years after diagnosis (that is, number of years lived with breast cancer). Details on the mortality data and the assumptions made in order to estimate mortality of women with and without breast cancer are reported in Supplementary File 3.

\section{Utility data}

To estimate total QALYs over lifetime for babies who did not die from infectious diseases or SIDS owing to the protective effect of breastfeeding, as well as for women without breast cancer, age- and gender-specific EQ-5D-derived utility values for the UK population were used [34] (Table 5).
Table 5 Utility values of the general UK population - EQ-5D ratings [34]

\begin{tabular}{lll}
\hline Age (years) & \multicolumn{2}{l}{ Utility mean (standard error) } \\
\cline { 2 - 3 } & Males & Females \\
\hline Under 25 & $0.94(0.01)$ & $0.94(0.01)$ \\
25 to 34 & $0.93(0.01)$ & $0.93(0.01)$ \\
35 to 44 & $0.91(0.01)$ & $0.91(0.01)$ \\
45 to 54 & $0.84(0.02)$ & $0.85(0.01)$ \\
55 to 64 & $0.78(0.02)$ & $0.81(0.02)$ \\
65 to 74 & $0.78(0.02)$ & $0.78(0.02)$ \\
$75+$ & $0.75(0.03)$ & $0.71(0.02)$ \\
\hline
\end{tabular}

Utility values for women with breast cancer were estimated based on data reported in a systematic review and meta-analysis of utility values for breast cancer [35], after taking into account the proportion of women with de novo stage IV (metastatic) disease among prevalent cases of women with metastatic breast cancer [36], and the percentage of women with breast cancer that have metastases at diagnosis [37]. Details on the utility data reported in the study and further assumptions made for estimation of QALYs are provided in Supplementary File 3.

\section{Cost data utilised in the model}

National sources were used to obtain the unit cost of a GP visit (£37) [16], as well as the cost of hospitalisation for GI ( $£ 756$ per admitted child) and for RTI ( $£ 1094$ per admitted child) [38]. The cost of death due to an infectious disease or SIDS per baby (£204) was also derived from national data [38] under the code VB99Z 'Emergency medicine, patient dead on arrival'. This was the only cost considered for mortality in babies in the basecase analysis.

Babies dying from an infectious disease are likely to have incurred further healthcare costs, some of which may have already been considered under other modelled clinical conditions and were therefore not considered in this part of the model. Post-mortem examination costs may also be attached to the death of a baby. Thus, a sensitivity analysis explored the impact on the results of adding a post-mortem examination cost of $£ 8000$ (paediatric coronial case plus forensic examination) [39] to each baby's death due to an infectious disease or SIDS. There are also considerable intangible emotional costs to parents following the death of a baby, which were not possible to include in the analysis.

Healthcare costs incurred by women with breast cancer and those without were obtained from a study on 359,771 
women with breast cancer in England [40]. Details on the cost data reported in that study and the assumptions used in order to utilise available data in our model are provided in Supplementary File 3.

\section{Discounting}

Where costs and/or outcomes were measured over a period longer than one year, they were discounted at an annual rate of $3.5 \%$ as recommended by NICE [41]. An annual discount rate of $1.5 \%$ was tested in sensitivity analysis, which is suggested by NICE for public health interventions [41].

\section{Analysis}

To account for the uncertainty around input parameter point estimates, a probabilistic analysis was undertaken, in which input parameters were assigned probabilistic distributions [42]. Subsequently, 10,000 iterations were performed, each drawing random values out of the distributions fitted onto the model input parameters. Mean costs and outcomes for each strategy were calculated by averaging across the 10,000 iterations. The incremental cost-effectiveness ratio (ICER) was calculated using the formula:

$$
\mathrm{ICER}=\Delta \mathrm{C} / \Delta \mathrm{E}
$$

where $\Delta \mathrm{C}$ is the difference in total costs between two treatment options considered and $\Delta \mathrm{E}$ the difference in their effectiveness (QALYs). The ICER expresses the extra cost per extra unit of benefit (QALY) associated with one treatment option relative to its comparator. If an option has an ICER of up to $£ 20,000-£ 30,000 / Q A L Y$ relative to its comparator (NICE lower and upper cost-effectiveness threshold, respectively) then the intervention is considered to be cost-effective [43].

Table 6 shows the deterministic values and probability distributions of all model input parameters and the methods employed to define their range. Deterministic values were used in a two-way sensitivity analysis, in which we changed concurrently the mean effect (RR) and cost of the intervention, to explore the impact of changes on the cost-effectiveness results. The ranges tested were from 1.05 to 2.00 for the intervention effect; and from $£ 20$ to $£ 100$ for the intervention cost.

In addition, one-way sensitivity analysis explored the following scenarios:

- Attaching a post-mortem cost of $£ 8000$ to each baby's death caused by an infectious disease or SIDS

- Assuming that women received their intervention when having their first baby and that the effect of the intervention was retained in future births, thus the benefits of breastfeeding applied to all future babies born by each woman receiving the intervention (in total 1.70 babies per woman [14]). For this scenario, we also assumed that the total duration of breastfeeding across pregnancies was 12 months; however, despite of the dose-response relation between breastfeeding duration and breast cancer risk in parous women, the effect of breastfeeding on the incidence of breast cancer was the same between a 6- and a 12-month duration [22]

- Use of an annual discount rate of $1.5 \%$

- Changing the starting age of the cohort of mothers by \pm 5 years ( 25 and 35 years of age).

\section{Validation of the economic model}

The economic model was developed in collaboration with members of the guideline committee, using a previous economic model [18] as a basis. All inputs and model formulae were systematically checked. The model was tested for logical consistency by setting input parameters to null and extreme values and examining whether results changed in the expected direction. Results were discussed with the committee to confirm their plausibility. Moreover, where modelling structure components were identical to those of Renfrew et al. [18], input data from that study were used to confirm that its results could be replicated using our model.

\section{Results}

Table 7 shows the results of the base-case economic analysis. The table provides the total intervention cost as well as total costs and outcomes associated with every clinical condition considered in the economic analysis, for 1000 women and their babies. The intervention had better outcomes and resulted in cost-savings across all conditions examined, when added on standard care compared with standard care alone. Overall, it was more costly than its comparator as the cost-savings resulting from provision of the intervention were not adequate to offset the intervention costs. The ICER of the intervention added on standard care versus standard care alone was £51,946/ QALY, which is well above the NICE upper cost-effectiveness threshold of $£ 30,000 / Q A L Y$ [43], suggesting that the intervention is not cost-effective. The table shows the results of the deterministic analysis, as these are directly comparable to the results of the two-way sensitivity analysis. Results of deterministic and probabilistic sensitivity analysis were very similar; the ICER of the probabilistic analysis was $£ 51,639 /$ QALY.

Results of two-way sensitivity analysis are shown in Table 8, for different combinations of intervention effect and intervention cost. Result cells with bold content 


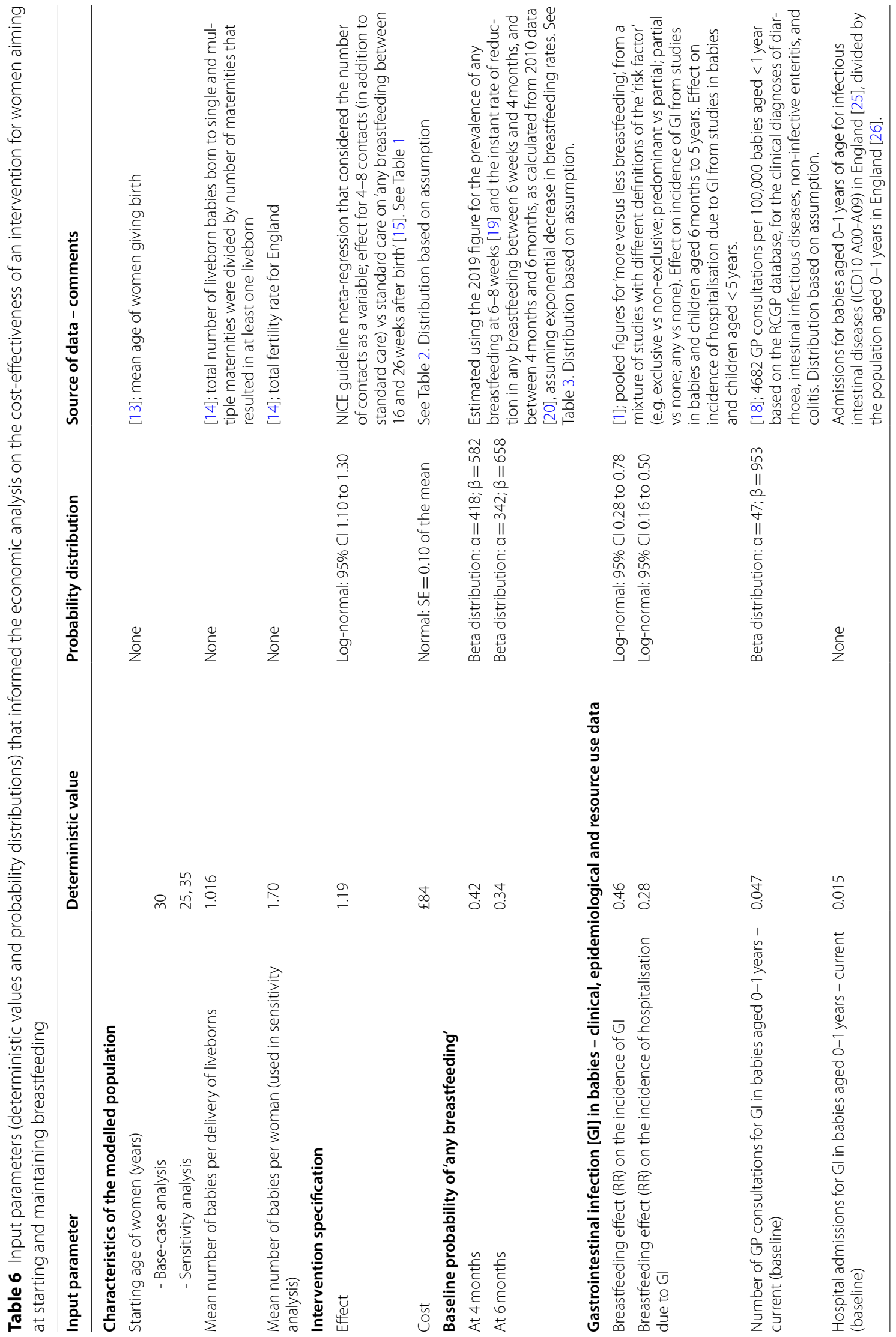




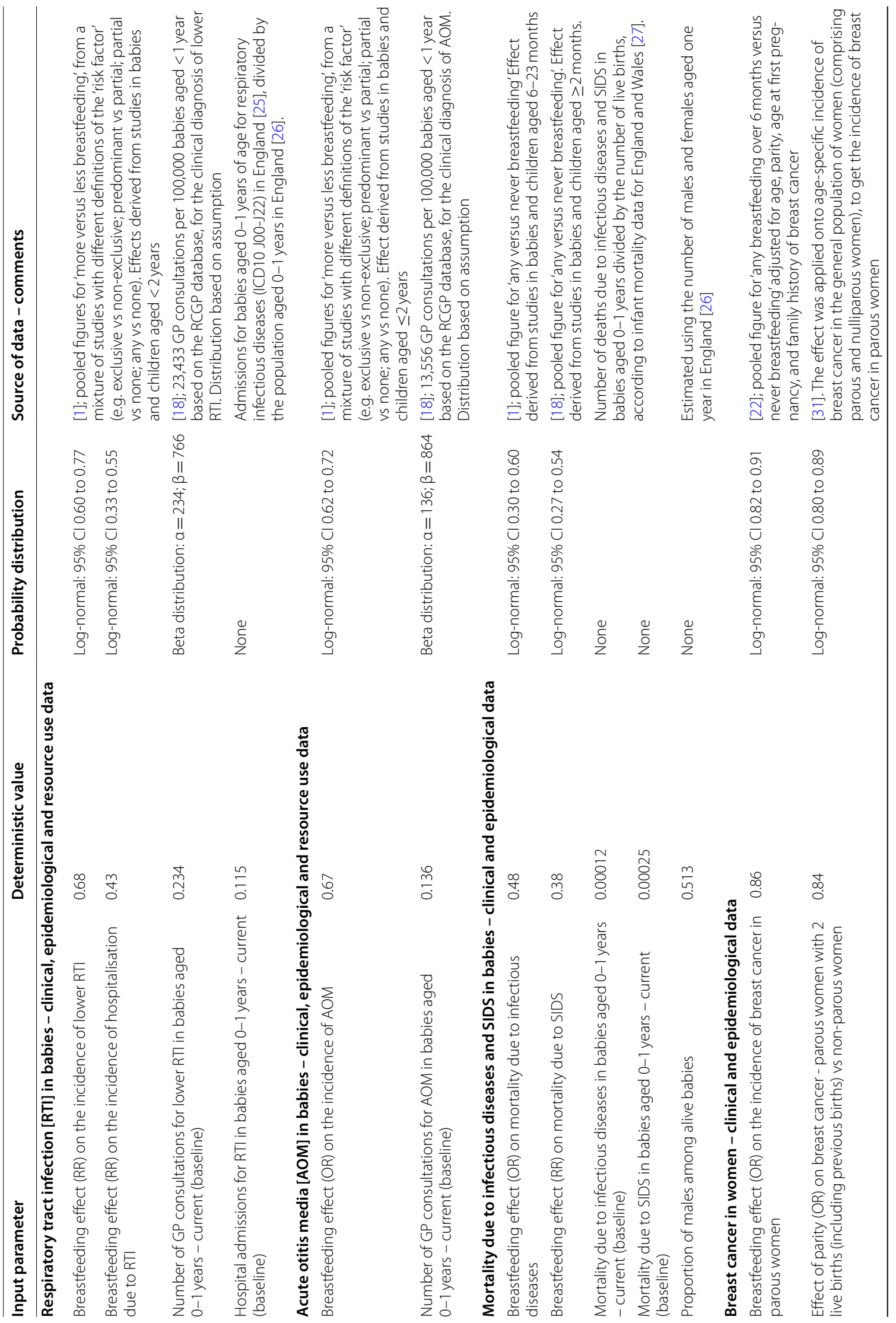




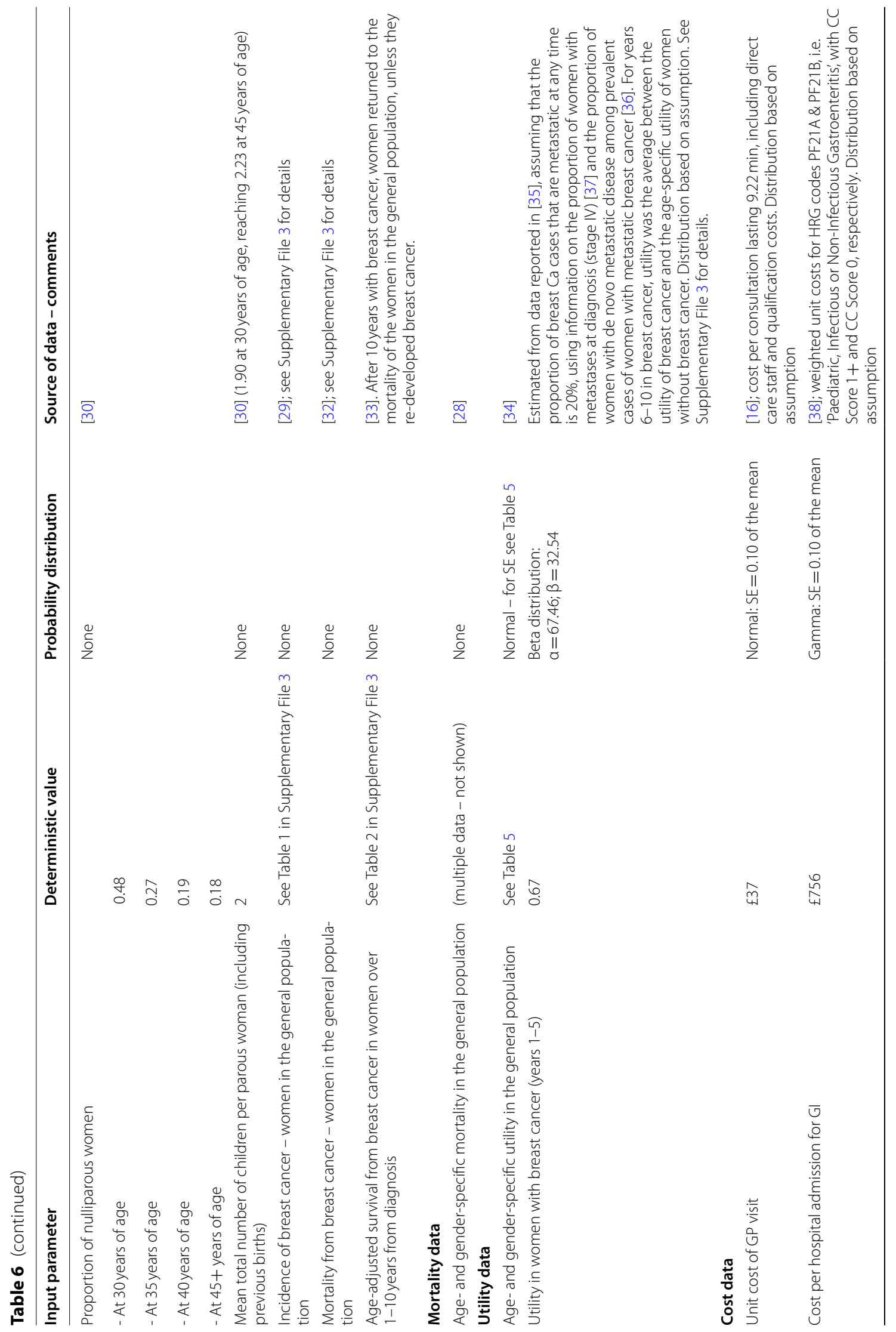




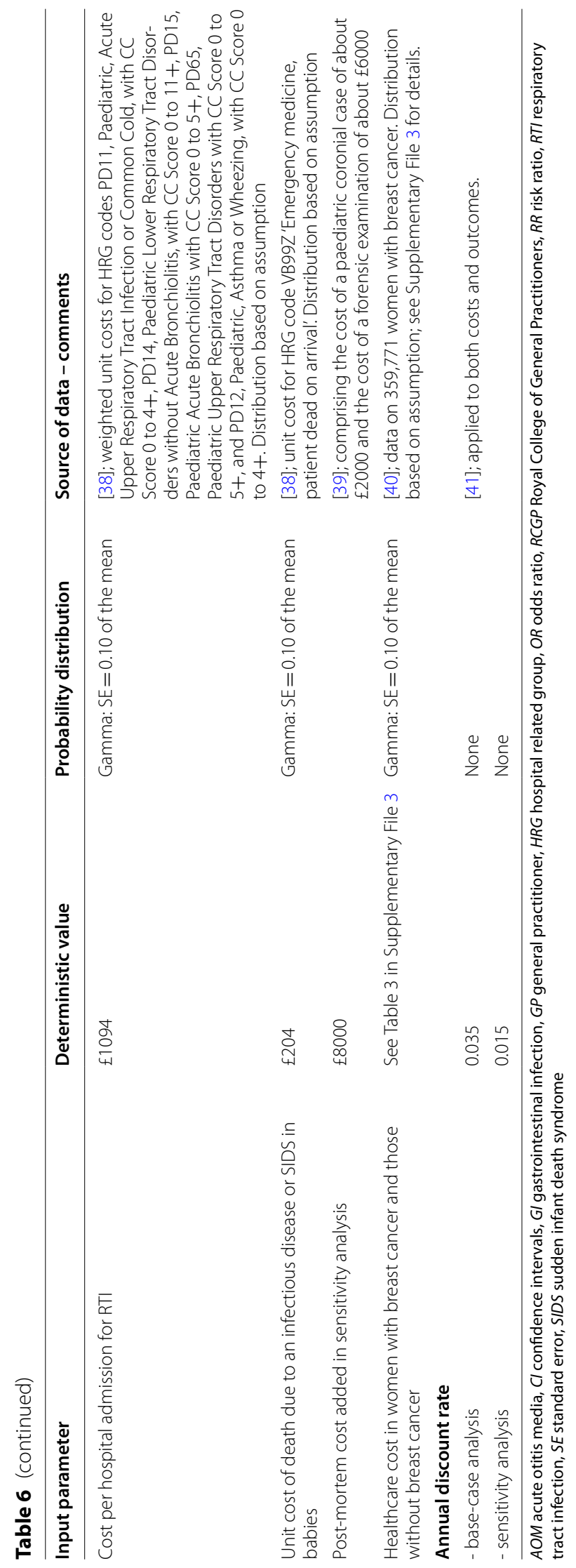


Table 7 Results of base-case economic analysis: cost-effectiveness of interventions aiming at promoting breastfeeding (results for 1000 women and their babies)

\begin{tabular}{|c|c|c|c|c|}
\hline Parameter & & Intervention $+\mathrm{SC}$ & SC alone & Difference \\
\hline Intervention cost & & $£ 84,000$ & $£ 0$ & $£ 84,000$ \\
\hline \multirow[t]{3}{*}{ Gl in babies } & Infections & 44.91 & 47.56 & -2.65 \\
\hline & Hospitalisations & 14.27 & 15.55 & -1.28 \\
\hline & Costs & $£ 12,469$ & $£ 13,535$ & $-£ 1066$ \\
\hline \multirow[t]{3}{*}{ (lower) RTI in babies } & Infections & 231.01 & 238.04 & -7.02 \\
\hline & Hospitalisations & 110.26 & 117.27 & -7.01 \\
\hline & Costs & $£ 129,272$ & $£ 137,204$ & $-£ 7932$ \\
\hline \multirow[t]{2}{*}{ AOM in babies } & Infections & 133.49 & 137.70 & -4.21 \\
\hline & Costs & $£ 4993$ & $£ 5150$ & $-£ 157$ \\
\hline \multirow{4}{*}{$\begin{array}{l}\text { Mortality in babies due to infections } \\
\text { and SIDS }\end{array}$} & Deaths due to infections & 0.11 & 0.12 & -0.01 \\
\hline & Deaths due to SIDS & 0.24 & 0.25 & -0.02 \\
\hline & Costs of deaths prevented & $-£ 1$ & & $-£ 1$ \\
\hline & QALYs gained & 0.16 & & 0.16 \\
\hline \multirow[t]{3}{*}{ Breast cancer in women } & New cases & 138.35 & 139.65 & -1.29 \\
\hline & QALYS & $20,945.72$ & $20,944.63$ & 1.09 \\
\hline & Costs & $£ 7,033,056$ & $£ 7,043,111$ & $-£ 10,056$ \\
\hline Total difference in QALYs & & & & 1.25 \\
\hline Total difference in costs & & & & $£ 64,787$ \\
\hline ICER & & $£ 51,946 / \mathrm{QALY}$ & & \\
\hline
\end{tabular}

AOM acute otitis media, GI gastrointestinal infection, ICER incremental cost-effectiveness ratio, RTI respiratory tract infection, SC standard care, SIDS sudden infant death syndrome

show combinations for which the intervention is costeffective, with an ICER below the NICE lower costeffectiveness threshold of $£ 20,000 / Q A L Y$, or with the intervention being dominant when added onto standard care, i.e. being both more effective and less costly compared with standard care alone. Result cells with figures in italics show combinations where the ICER is between $£ 20,000-£ 30,000$ /QALY. All other result cells show combinations for which the intervention is not cost-effective, with an ICER above the NICE upper cost-effectiveness threshold of $£ 30,000 / Q A L Y$. The underlined figures show the intervention cost and effect values used in base-case analysis and the base-case ICER.

As expected, the cost-effectiveness of the intervention improves as its effectiveness increases and its intervention cost decreases. At the base-case relative effect (RR) of 1.19 (for any breastfeeding at 16-26 weeks after birth), the intervention becomes cost-effective $(<£ 20,000 /$ QALY) if its cost per woman receiving the intervention is approximately $£ 40-£ 45$. On the other hand, at the basecase cost of $£ 84$, the intervention becomes cost-effective if its effectiveness (in terms of breastfeeding rates), when added on standard care, is at least $35-40 \%$ higher than the effectiveness of standard care alone (i.e. if the $R R$ reaches $1.35-1.40)$.
Table 9 shows results of other scenarios tested in sensitivity analysis. Inclusion of the post-mortem examination cost for babies dying due to an infectious disease or SIDS had practically no impact on the ICER, which was expected, given the very low mortality due to an infectious disease or SIDS in babies aged 0-1years. Similarly, assuming that all women received the intervention when they had their first baby and that the effect of the intervention on breastfeeding rates would be retained in all subsequent births had no impact on our conclusion. On the other hand, results were considerably affected by the use of an annual discount rate of $1.5 \%$ for both costs and outcomes, with the ICER falling at a value between the lower and upper NICE cost-effectiveness threshold of $£ 20,000-£ 30,000 /$ QALY. This finding is explained by the fact that the greatest part of clinical benefits of breastfeeding (prevention of breast cancer) is enjoyed by women several years after breastfeeding takes place (as the incidence of breast cancer in women considerably increases after the age of 40 years), so reducing the discount factor places a greater value on the benefits and cost-savings accrued in the long-term. Because of discounting, reducing the starting age of women cohort at 25 years increased the ICER (as QALY gains resulting from prevention of breast cancer occurred after a longer 


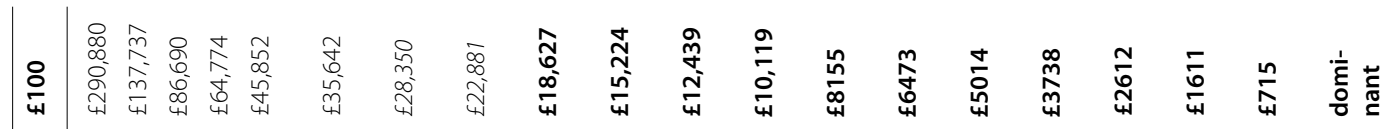

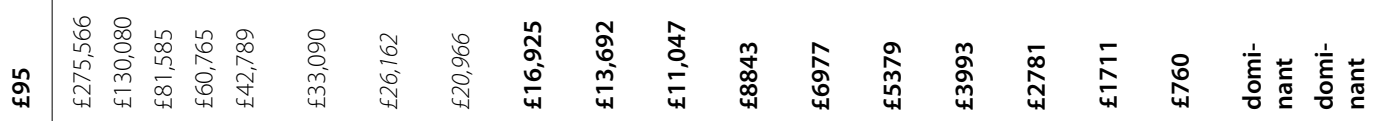

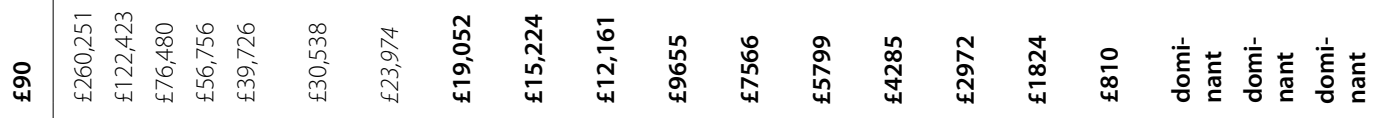

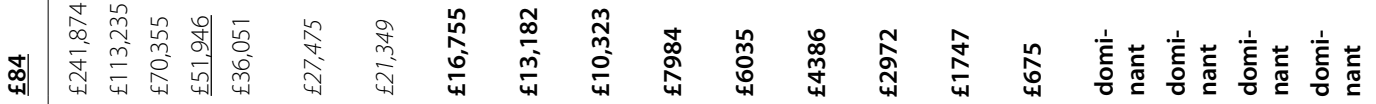

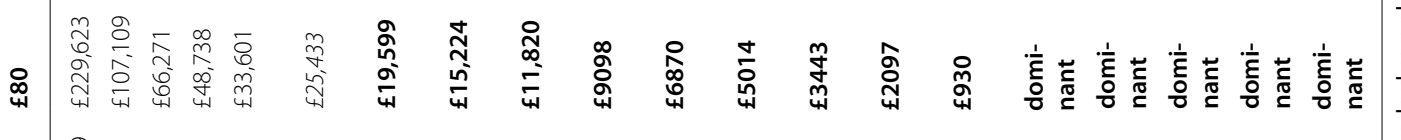

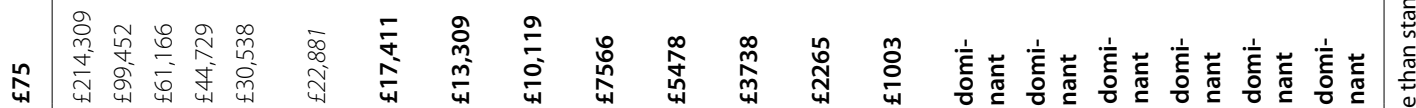

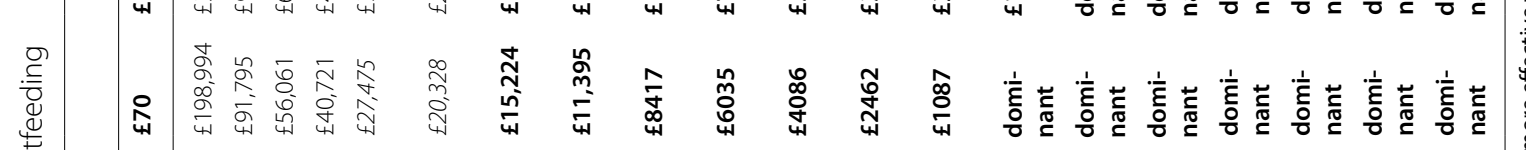

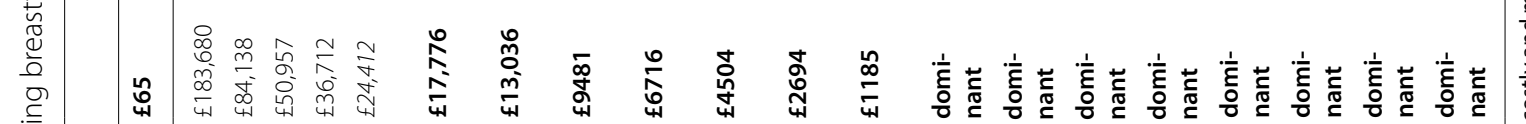

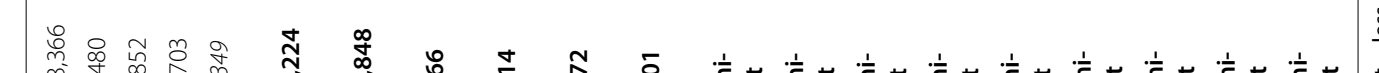

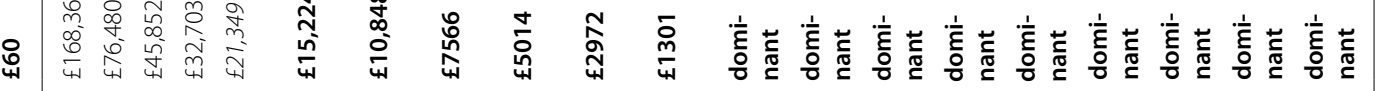

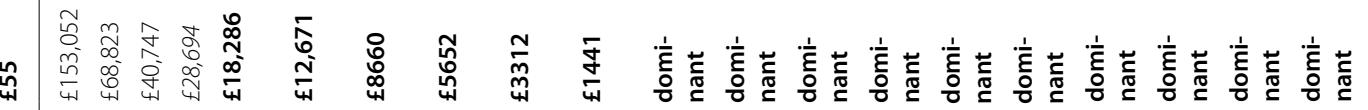

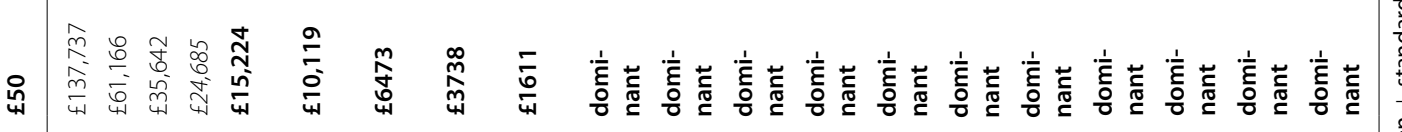

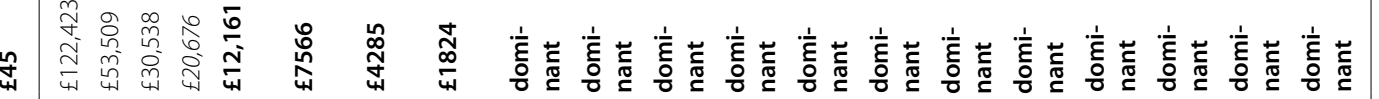

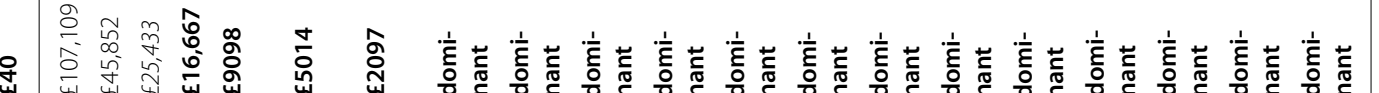

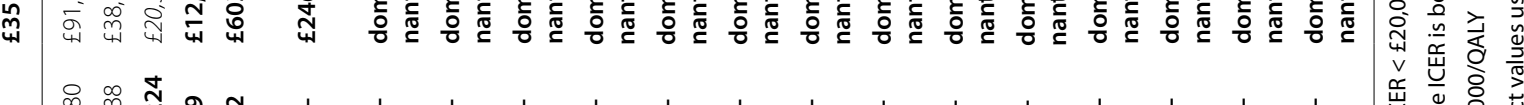

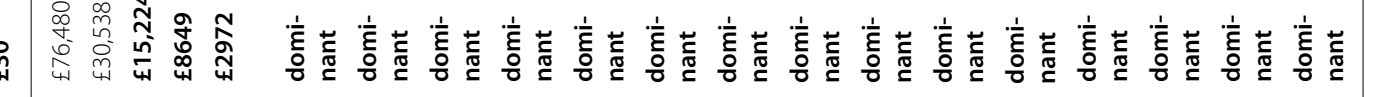

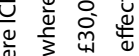

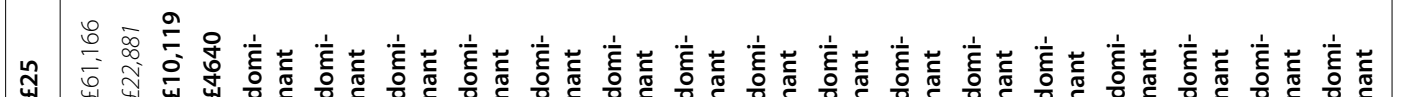
莺

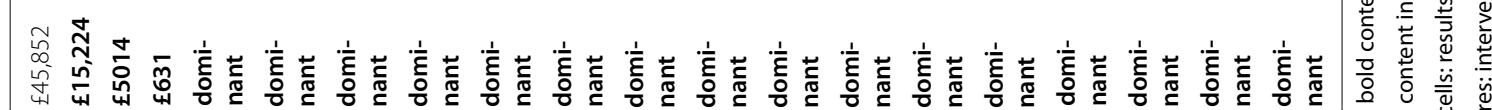

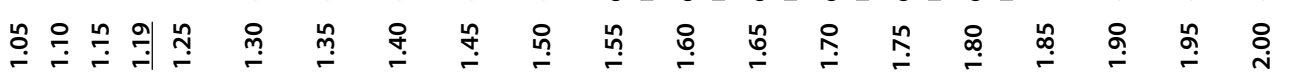

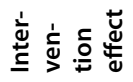


Table 9 Results of alternative scenarios tested in sensitivity analysis

\begin{tabular}{ll}
\hline Scenario tested in sensitivity analysis & ICER \\
\hline $\begin{array}{l}\text { Inclusion of post-mortem examination cost for baby } \\
\text { deaths }\end{array}$ & $£ 51,904 / \mathrm{QALY}$ \\
$\begin{array}{ll}\text { Assuming effect of intervention is retained in future } \\
\text { births, so that breastfeeding benefits apply to all babies }\end{array}$ & $£ 43,223 / \mathrm{QALY}$ \\
born to a woman & \\
Use of an annual discount rate of 1.5\% & $£ 22,667 / \mathrm{QALY}$ \\
Starting age of women 25years & $£ 60,145 / \mathrm{QLAY}$ \\
Starting age of women 35years & $£ 46,068 / \mathrm{QLAY}$ \\
\hline
\end{tabular}

period of time compared with the base-case analysis and were thus placed a lower value due to discounting), whereas increasing the starting age of women at 35 years reduced the ICER (because prevention of breast cancer occurred sooner compared with the base-case analysis and was thus placed a higher value). Since women aged $\leq 35$ years represent $78 \%$ of women giving birth in England and Wales [13], the intervention is unlikely to be cost-effective under NICE criteria for the whole study population, even if it is found to be cost-effective in women giving birth aged beyond 35 years.

\section{Discussion}

\section{Overview and interpretation of findings}

The results of our analysis suggest that an intervention aimed at promoting breastfeeding, which comprises provision of information and support to women by healthcare workers and/or breastfeeding peer supporters, initiated in the antenatal period or up to 8 weeks after birth, is unlikely to be cost-effective, as its ICER when added onto standard care versus standard care alone was $£ 51,946 /$ QALY, which is well above the NICE upper cost-effectiveness threshold of $£ 30,000 /$ QALY. Results of sensitivity analysis suggested that the intervention might become cost-effective if its cost was reduced by about $50 \%$ (from $£ 84$ to around $£ 40-£ 45$ per woman receiving the intervention) or if its relative effect (RR) on any breastfeeding 16-26 weeks after birth was improved by about $100 \%$ (from 1.19, i.e. $19 \%$ increase, to $1.35-1.40$, i.e. $35-40 \%$ increase in breastfeeding rates). Translating the intervention effect into numbers of women breastfeeding, in a cohort of 100 women, 42 would breastfeed under standard care (estimate based on national statistics), 50 would breastfeed if the modelled intervention with a RR of 1.19 was offered to the cohort, and 57 women in the cohort would need to breastfeed (estimated using a RR of 1.36) for the intervention to become cost-effective.

A less resource intensive intervention comprising two individual 30-min sessions provided by a health professional in NHS England AfC Band 5 has a cost of $£ 59$, which is higher than the cost of $£ 40-45$ that would be required for the intervention to be cost-effective; moreover, according to our meta-regression, an intervention comprising two contacts would only be expected to have a small and non-significant effect (RR 1.07, 95\% CI 0.98 to 1.17 , for $2-3$ contacts versus standard care). An intervention cost of $£ 40$, required for the intervention to become cost-effective, could be achieved by 4 individual 30-min sessions provided by a peer supporter, assuming a unit cost of $£ 20$ per hour. However, it is possible that the unit cost of a peer supporter is higher, if childcare costs are taken into account, meaning that an intervention cost as low as $£ 40$ may not be achievable even by provision of the intervention by a peer supporter offering 4 individual 30-min sessions.

Furthermore, the RR of 1.35-1.40 that would be required for the intervention to become cost-effective is above the upper $95 \% \mathrm{CI}$ of the relative effect used in the base-case analysis (mean RR $1.19,95 \%$ CI 1.10 to 1.30 ). Therefore, it appears that an intervention of this type needs to be both more effective and less costly than its specification in our economic analysis, for it to be costeffective within the NICE decision-making context.

\section{Strengths and limitations}

The effectiveness of the intervention in improving breastfeeding rates was determined by a meta-regression of RCTs [15] conducted to inform the NICE guideline on postnatal care [12]. The quality of the included data was overall low. Typically, the studies included in the review were characterised by serious risk of bias associated with the randomisation process, selective reporting and missing outcome data, as assessed using the Cochrane Risk of Bias tool [44]. Most studies were unblinded, which was unsurprising given the nature of the interventions. Interventions included in the review were characterised by particularly high heterogeneity regarding the mode of delivery (e.g. face-to-face or by telephone, individually or in groups, blended interventions were also common), the number of contacts and the duration of the intervention, the place of delivery (at home, in hospital, in a community setting or a combination of locations), the person delivering the intervention (peer supporter, lactation consultant, midwife, health visitor or a combination), and the involvement of fathers. Moreover, there was heterogeneity regarding the study participants' intention to breastfeed at recruitment, and the definition of 'any breastfeeding' as an outcome. Standard care was also heterogeneous and, in general, poorly described.

The structure of the economic model and the estimates of the association of breastfeeding with clinical outcomes were based on high quality studies $[1,18]$ 
identified from a systematic review of the literature conducted specifically to inform our analysis. However, the primary data on the association of breastfeeding with clinical outcomes that were synthesised in meta-analysis were derived from study designs that were prone to bias; several studies demonstrating clinical benefits associated with breastfeeding had adjusted for some known confounders. Nevertheless, it is possible that there are other unknown confounders impacting on the relation between breastfeeding and clinical benefits, for which the studies were unable to adjust. Moreover, other studies made no adjustments for confounding. Thus, it is possible that the magnitude of the clinical benefits of breastfeeding have been overestimated in this literature and, consequently, in our analysis.

One further point to note is that evidence on the association between breastfeeding and mortality from infectious diseases was derived exclusively from low and medium income countries due to lack of high income country data, so findings may not be directly relevant to the population in the UK. However, the impact of this input parameter on the results was negligible.

Breastfeeding has been found to be associated with several clinical conditions that were not possible to consider in our economic model, either due to lack of suitable and/or good quality epidemiological and cost data that would allow robust modelling to be conducted, or due to the complexity or uncertainty of modelling owing to the multifactorial nature of some diseases. For example, breastfeeding has been associated with a reduced risk of diabetes in both mothers and babies and a reduced risk of obesity in babies over their lifetime. It has also been associated with improved cognitive outcomes in babies and reduced incidence of ovarian cancer in mothers [1]. Furthermore, there is some evidence that breastfeeding has a protective effect on the development of triple negative breast cancer $[45,46]$, which is considered to be more aggressive and have a poorer prognosis compared with other types of breast cancer. Current evidence suggests a protective effect of breastfeeding on the incidence of and hospitalisations due to GI in babies over the first 5 years of their life, and on the incidence of lower RTI and AOM, as well as hospitalisations due to RTI over the first 2 years of their life [1]. We conservatively modelled respective cost-savings and benefits to babies only over the first year of their lives, due to the availability of relevant epidemiological data. Prevention of infections in babies, which is associated with breastfeeding, results in lower antibiotic use and thus lower rates of antimicrobial resistance in the community. Mothers who wish to breastfeed but experience societal barriers or lack of skilled support may experience psychological distress if they are not able to breastfeed. A successful breastfeeding intervention that enables them to breastfeed is likely to improve their mental wellbeing and promote emotional attachment with their baby, improving also the baby's psychological development. Such benefits were not captured in our analysis.

Clinical benefits such as the reduction in the incidence of GI, RTI and AOM in babies were not translated into QALYs and thus were not considered in the estimation of the ICER. On the other hand, QALY gains associated with these benefits are expected to be very small due to the usually short duration of these outcomes (only a few days or weeks). Equally, the ICER has not captured the intangible benefits to parents associated with improved outcomes in babies, in particular the psychological burden avoided by a reduction in mortality due to infectious diseases or SIDS.

Benefits were only estimated for healthy babies at term. Breastfeeding has been shown to be associated with clinical benefits to premature, low-birth-weight, or seriously ill babies [47-50]. Such benefits were not captured by our analysis as they were beyond its scope.

The clinical data on the protective effect of breastfeeding on the incidence of breast cancer expressed the difference in the incidence of breast cancer between parous women that breastfed over 6 months after birth and those who never breastfed [22]; similarly, the clinical data on the protective effect of breastfeeding on mortality due to infectious diseases or SIDS in babies expressed the difference in mortality between babies that were breastfed (at 4 months after birth) and those that were never breastfed. A shorter duration of breastfeeding (e.g. $2-3$ months) is possible to still have a protective effect on breast cancer in women [22] and death from infectious diseases or SIDS in babies. However, our model considered the effectiveness of the breastfeeding intervention regarding the breastfeeding status of women and babies at a single time point ( 4 months after birth for babies, 6 months for women). Some of the women who were not breastfeeding at 6 months may have breastfed until an earlier time point (i.e. they are not necessarily women who never breastfed their babies from birth to 6 months); similarly, some of the babies who were not breastfed at 4 months may have been breastfed until an earlier time point (i.e. they are not necessarily babies who were never breastfed from birth and up to 4 months); these women and babies have received some protective effect from breastfeeding in reducing the incidence of breast cancer and mortality due to infectious diseases or SIDS, respectively. In this aspect, our analysis has likely overestimated the benefits and cost-savings of breastfeeding (and, consequently, of the breastfeeding intervention) to mothers and babies. 
As infant mortality from infectious diseases or SIDS is rare, the overestimation of the protective effect of breastfeeding is expected to be negligible and thus highly unlikely to have impacted on the results and conclusions of the analysis. In contrast, the overestimation of the protective effect of breastfeeding on the reduction in the incidence of breast cancer has potentially a significant impact on the model results, given that the QALYs and cost-savings from the reduction in the incidence of breast cancer contributed considerably to the estimation of the ICER (QALYs gained due to a reduction in the incidence of breast cancer accounted for 95\% of total QALYs gained following provision of the breastfeeding intervention; cost-savings due to a reduction in the incidence of breast cancer accounted for $51 \%$ of the total cost-savings following provision of the breastfeeding intervention).

According to an older high-quality meta-analysis of 47 epidemiological studies in 30 countries [17], the impact of any versus no breastfeeding for up to 6 months on breast cancer is very small and non-significant (OR 0.98, $95 \%$ CI 0.95 to 1.01 ), while the impact of any versus no breastfeeding for a duration of 7-18 months is statistically significant (OR $0.94,95 \%$ CI 0.91 to 0.97 ), but smaller than the estimate from the meta-analysis that informed our economic analysis [22]. The two metaanalyses included different studies and reported rather contradictory results on whether breastfeeding for a duration of up to 6 months can reduce the incidence of breast cancer. Results of the older meta-analysis also suggest that our model may have overestimated the clinical benefits and associated cost-savings of the breastfeeding intervention in relation to the reduction in the incidence of breast cancer. It is noted, though, that Victora et al. [1] reported the results of a meta-analysis, according to which highest versus lowest duration of breastfeeding showed a larger and statistically significant protective effect on the incidence of breast cancer (OR 0.81, 95\% CI 0.77 to 0.86 ). However, most studies in this meta-analysis had not adjusted appropriately for parity and therefore tended to exaggerate the effect size.

The guideline committee expressed the view that, overall, the magnitude of overestimation of benefits and cost-savings in some aspects of the economic analysis balanced out the magnitude of the underestimation of benefits and cost-savings in other areas, where modelling was limited or not conducted. After considering the strengths and the weaknesses of our analysis, the committee concluded that, currently, providing an education and support intervention, in addition to standard care, that aims to promote breastfeeding to women that are pregnant or have given birth to healthy babies at term, does not appear to be cost-effective in the UK under NICE criteria of cost-effectiveness.
Generalisability of the results and implications of the study Our analysis was conducted from the perspective of the NHS/PSS in England. Results may be generalisable to other settings with similar funding and structure of healthcare and personal social services, including postnatal care pathways, as well as comparable epidemiological picture regarding the clinical conditions associated with breastfeeding. Interventions for mothers, aimed at promoting breastfeeding, may be considerably more cost-effective in settings where the baseline risk of infections to babies and/or breast cancer to mothers, and/or associated management costs, are higher compared with the UK, for example, in low and middle-income country settings. We also note that our results were sensitive to the discount rate used, as use of a lower discount rate led to the intervention's cost-effectiveness being considerably improved. The choice of the discount rate for costs and outcomes in economic evaluations of health care programmes and policies has been contentious and the subject of ongoing discussion [51-54] and may have a stronger impact in economic evaluations of strategies with long-term consequences, such as prevention programmes, which are disfavoured by use of higher discount rates $[52,53]$. Conclusions on cost-effectiveness ultimately rely on the perspective of the analysis and the cost-effectiveness threshold adopted; the latter depends on the policy makers' willingness-to-pay for clinical and other wider benefits, which may vary across countries and health systems.

As other literature suggests, worldwide, breastfeeding itself is cost-effective as it leads to important clinical benefits to mothers and babies and cost-savings to the health service, parents and the whole society, at no intervention cost $[18,21,55-60]$. Our economic analysis only demonstrated that the breastfeeding intervention, as specified in our model, was not cost-effective when added to standard care because the clinical benefits and cost-savings resulting from an increase in breastfeeding rates, although important, were not adequate to outweigh initial intervention costs. This is because the baseline incidence of the clinical conditions assessed in the model is already rather low in the general population of women giving birth to healthy babies, and their babies, in the UK compared with other settings, e.g. the rate of baby infections is higher in low-income countries and therefore clinical benefits to babies and associated cost-savings resulting from breastfeeding (i.e. a reduction in baby infection rates and subsequent hospitalisations) are expected to be larger. Moreover, the effectiveness of the intervention in improving breastfeeding rates at 16-26 weeks was relatively small (mean RR of the intervention added onto standard care versus standard care alone 1.19), with a rather small impact at a population level. To put 
this figure into context, out of 100 women receiving the intervention, 42 would breastfeed at 4 months under standard care whether they received the intervention or not (according to national statistics), and only 8 would breastfeed because of the intervention (19\% of 42 according to the intervention's effectiveness). Therefore, the benefit of the intervention offered to 100 women would be 8 additional women breastfeeding. At an intervention cost of $£ 84$ per woman receiving the intervention, it would cost $£ 8400$ to support 8 extra women to breastfeed, or $£ 1050$ per extra woman breastfeeding.

Future research should focus on the effective components of breastfeeding interventions, as identified by this meta-regression, aiming at the development of breastfeeding interventions with higher effectiveness that are less resource intensive and offer more return on investment than currently available interventions. Women's needs and views on facilitators and barriers to breastfeeding need also to be taken into account when designing such interventions.

Perhaps the way forward to improve breastfeeding rates in a cost-effective way is to implement public health and other societal interventions, which do not target women specifically but apply changes within society as a whole to promote and normalise breastfeeding, after identifying and targeting factors that dissuade mothers from breastfeeding in current UK society, for example, the pressures of advertising by infant formula companies, negative public attitudes to breastfeeding in public, and the lack of appropriate facilities for breastfeeding mothers. Such interventions could include, for example, full adoption of the WHO International Code of marketing of breast milk substitutes [61], further improvements to maternity leave arrangements and pay, and other workplace policies that enable working women to breastfeed their babies. Such interventions were beyond the scope of the NICE updated guideline on postnatal care, and therefore we have not explored their effects and cost-effectiveness. It is also possible that, if some of the barriers to breastfeeding are removed by implementation of societal interventions, this will lead to an improvement of the effectiveness of education and support interventions targeted to pregnant women and those who have given birth.

Based on the results of this meta-regression and economic analysis, the guideline committee was unable to recommend specific education and support breastfeeding interventions of the type assessed in the economic analysis; instead, they made recommendations that aim to enforce national breastfeeding initiatives and optimise current NHS postnatal care in England, to improve the quality and reduce variation in the current provision of breastfeeding advice and support across settings.

\section{Conclusion}

Worldwide, breastfeeding results in health benefits to women and their babies and cost-savings to the health service. Available evidence on antenatal and postnatal breastfeeding interventions for women, which comprise education, advice and support from a mixture of health professionals and peer volunteers, suggests these may not be cost-effective when added to standard care under NICE criteria in England. More effort needs to be placed on how breastfeeding education, advice and support can be optimised in order to improve the quality and reduce variation in services offered across care settings. Public health and other societal interventions that facilitate breastfeeding and remove barriers to it may be key in improving breastfeeding rates in the UK.

\section{Abbreviations \\ AfC: Agenda for change; AOM: Acute otitis media; Cl: Confidence intervals; Gl: Gastrointestinal infection; GP: General practitioner; ICER: Incremental cost- effectiveness ratio; NHS: National health service; NICE: National Institute for Health and Care Excellence; OR: Odds ratio; PSS: Personal social services; QALY: Quality-adjusted life year; RCGP: Royal College of General Practitioners; RCT: Randomised controlled trial; RR: Risk ratio; RTI: Respiratory tract infection; SIDS: Sudden infant death syndrome; WHO: World Health Organization.}

\section{Supplementary Information}

The online version contains supplementary material available at https://doi. org/10.1186/s12889-021-12446-5.

Additional file 1. Systematic review of studies that modelled long-term clinical benefits to mothers and babies (and/or related cost-savings to health and personal social services) associated with breastfeeding: included studies and studies excluded after full text was obtained.

Additional file 2. Evidence on clinical conditions associated with breastfeeding that was considered in the development of the economic analysis on the cost-effectiveness of breastfeeding interventions.

Additional file 3. Age-specific incidence of breast cancer, mortality, cost and utility data that informed the economic model component on women's breast cancer.

\section{Acknowledgements}

We thank other members of the Guideline Committee for the NICE guideline on 'Postnatal care' for their contributions to this work. Members of the Guideline Committee were: David Jewell (chair), Nina Khazaezadeh (topic advisor), Gwyneth Eanor, Peter Fleming, Debra Kroll, Clare Macdonald, Denise Pemberton, Catherine Pullan, Elizabeth Punter, Sunita Sharma, Emily Stow, Charlotte Barry (co-opted member), Naomi Cotton (co-opted member), Lucinda Donaldson (co-opted member), Nicola Murphy (co-opted member), Deepa Panjwani (co-opted member). We also thank Rami Cosulich for her contribution to the systematic review of breastfeeding interventions, regarding study selection and data extraction.

\section{Authors' contributions}

IM conceptualised the study, developed the study design and constructed the economic model in Excel 2013. SS conducted all literature searches. JVC performed the literature review of efficacy of breastfeeding interventions, including the related data collection and statistical analysis. IM performed the literature review of costs and benefits of breastfeeding, including the related data collection and statistical analysis, and conducted the economic analysis. 
All authors were involved in the validation of the economic model structure, the data and the analysis, as well as the interpretation of the findings. IM wrote the first draft of the manuscript and all other authors were involved in reviewing and revising it. All authors read and approved the final manuscript.

\section{Funding}

This work was undertaken by the National Guideline Alliance (NGA) at the Royal College of Obstetricians and Gynaecologists (RCOG), which receives funding from the National Institute for Health and Care Excellence (NICE) in England to develop clinical, public health and social care guidelines. The views expressed in this publication are those of the authors and not necessarily those of the NGA, RCOG or NICE. The funder of the study (NICE) had no further role in the study design, data collection, data analysis, data interpretation, the writing of the report or the decision to submit this article for publication. All authors had full access to the study data and had final responsibility for the decision to submit for publication. National Institute for Health and Care Excellence (2021) Postnatal Care. Available from: https://www.nice.org.uk/guidance/ng194.

\section{Availability of data and materials}

All data generated or analysed during this study are included in this published article and its supplementary information files.

\section{Declarations}

\section{Ethics approval and consent to participate}

Not applicable.

\section{Consent for publication}

Not applicable.

\section{Competing interests}

IM, JVC, SSt, JF and MK received support from the National Guideline Alliance (NGA), which was in receipt of funding from the National Institute for Health and Care Excellence (NICE), for the submitted work. CM, SSh, PF, EP, CB, NK and DJ declared the following interests based on NICE's policy on conflicts of interests: https://www.nice.org.uk/guidance/ng194/documents/register-of-inter ests. The authors report no other relationships or activities that could appear to have influenced the submitted work.

\section{Author details}

${ }^{1}$ Centre for Outcomes Research and Effectiveness, Research Department of Clinical, Educational \& Health Psychology, University College London, 1-19 Torrington Place, London WC1E 7HB, UK. ${ }^{2}$ National Guideline Alliance, Royal College of Obstetricians and Gynaecologists, London, UK. ${ }^{3}$ Central Surgery, Oadby, Leicestershire, UK. ${ }^{4}$ Institute of Applied Health Research, University of Birmingham, Birmingham, UK. ${ }^{5}$ Chelsea and Westminster Hospital NHS Foundation Trust, London, UK. ${ }^{6}$ Centre for Academic Child Health, Bristol Medical School, University of Bristol, Bristol, UK. ${ }^{7}$ The Dudley group NHS foundation Trust, Dudley, West Midlands, UK. ${ }^{8}$ Midlands Maternity and Perinatal Mental Health Clinical Network, NHS England and NHS Improvement, London, UK. ${ }^{9}$ Guy's \& St. Thomas NHS Foundation Trust, London, UK. ${ }^{10}$ Chair of the committee responsible for the NICE guideline on postnatal care and General Practitioner, Bristol, UK.

\section{Received: 16 June 2021 Accepted: 20 December 2021}

Published online: 22 January 2022

\section{References}

1. Victora CG, Bahl R, Barros AJ, Franca GV, Horton S, Krasevec J, et al. Breastfeeding in the 21st century: epidemiology, mechanisms, and lifelong effect. Lancet. 2016;387(10017):475-90.

2. World Health Organization: breastfeeding in the 21 st century. WHO; 2017. https://www.who.int/pmnch/media/news/2016/breastfeeding_brief.pdf. Accessed 23 March 2021.

3. Unicef UK: Removing the barriers to breastfeeding: a call to action. Unicef UK; 2017. https://www.unicef.org.uk/babyfriendly/wp-content/uploads/ sites/2/2017/07/Barriers-to-Breastfeeding-Briefing-The-Baby-Friendly-Initi ative.pdf Accessed 23 Mar 2021.
4. Buckland C, Hector D, Kolt GS, Fahey P, Arora A. Interventions to promote exclusive breastfeeding among young mothers: a systematic review and meta-analysis. Int Breastfeed J. 2020;15(1):102.

5. Gomez L, Verd S, de-la Banda G, Cardo E, Servera M, Filgueira A, et al. Perinatal psychological interventions to promote breastfeeding: a narrative review. Int Breastfeed J. 2021;16(1):8.

6. Haroon S, Das JK, Salam RA, Imdad A, Bhutta ZA. Breastfeeding promotion interventions and breastfeeding practices: a systematic review. BMC Public Health. 2013;13(Suppl 3):S20.

7. Anokye N, Coyle K, Relton C, Walters S, Strong M, Fox-Rushby J. Costeffectiveness of offering an area-level financial incentive on breast feeding: a within-cluster randomised controlled trial analysis. Arch Dis Child. 2020;105(2):155-9.

8. Frick KD, Pugh LC, Milligan RA. Costs related to promoting breastfeeding among urban low-income women. J Obstet Gynecol Neonatal Nurs. 2012;41(1):144-50.

9. Hoddinott P, Britten J, Pill R. Why do interventions work in some places and not others: a breastfeeding support group trial. Soc Sci Med. 2010;70(5):769-78.

10. Hoddinott P, Britten J, Prescott GJ, Tappin D, Ludbrook A, Godden DJ. Effectiveness of policy to provide breastfeeding groups (BIG) for pregnant and breastfeeding mothers in primary care: cluster randomised controlled trial. BMJ. 2009;338:a3026.

11. Stevens B, Guerriere D, McKeever P, Croxford R, Miller KL, Watson-MacDonell J, et al. Economics of home vs. hospital breastfeeding support for newborns. J Adv Nurs. 2006;53(2):233-43.

12. National Institute for health and care excellence: postnatal care. NICE; 2021. https://www.nice.org.uk/guidance/ng194. Accessed 20 Apr 2021.

13. Office for National Statistics: Births by parents' characteristics, England and Wales 2017. Office for National Statistics; 2019. https://www.ons.gov.uk/ peoplepopulationandcommunity/birthsdeathsandmarriages/livebirths/ datasets/birthsbyparentscharacteristics. Accessed 13 November 2019.

14. Office for National Statistics: Birth characteristics in England and Wales: 2018. Office for National Statistics; 2019. https://www.ons.gov.uk/peopl epopulationandcommunity/birthsdeathsandmarriages/livebirths/bulle tins/birthcharacteristicsinenglandandwales/2018. Accessed 21 January 2020.

15. National Institute for health and care excellence: postnatal care. Evidence review P. Breastfeeding interventions. NICE; 2021. https://www.nice.org. uk/guidance/ng194/evidence/p-breastfeeding-interventions-pdf-32676 4485980 Accessed 20 Apr 2021.

16. Curtis L, Burns A. Unit costs of Health \& Social Care 2018. Canterbury: PSSRU, University of Kent; 2018.

17. Collaborative group on hormonal factors in breast cancer. Breast cancer and breastfeeding: collaborative reanalysis of individual data from 47 epidemiological studies in 30 countries, including 50302 women with breast cancer and 96973 women without the disease. Lancet. 2002;360(9328):187-95.

18. Renfrew MJ, Pokhrel S, Quigley M, McCormick F, Fox-Rushby J, Dodds R, et al. Preventing disease and saving resources: the potential contribution of increasing breastfeeding rates in the UK. Commissioned by UNICEF UK; 2012. https://www.unicef.org.uk/babyfriendly/wp-content/uploads/ sites/2/2012/11/Preventing_disease_saving_resources.pdf. Accessed 14 November 2019.

19. Public Health England: Statistical release: breastfeeding at 6 to 8 weeks, 2018 to 2019 annual (November 2019 release). PHE; 2019. https://www. gov.uk/government/statistics/breastfeeding-at-6-to-8-weeks-after-birthannual-data. Accessed 20 November 2019.

20. NHS Digital: Infant feeding survey - UK, 2010. Prevalence of breastfeeding at different ages. NHS Digital; 2012. https://digital.nhs.uk/data-and-infor mation/publications/statistical/infant-feeding-survey/infant-feedingsurvey-uk-2010. Accessed 20 November 2019.

21. Unar-Munguia M, Meza R, Colchero MA, Torres-Mejia G, Gonzalez de Cosio T. Economic and disease burden of breast cancer associated with suboptimal breastfeeding practices in Mexico. Cancer Causes Control. 2017;28(12):1381-91.

22. Unar-Munguia M, Torres-Mejia G, Colchero MA, Gonzalez de Cosio T. Breastfeeding mode and risk of breast Cancer: a dose-response Metaanalysis. J Hum Lact. 2017;33(2):422-34.

23. Bartick M, Reinhold A. The burden of suboptimal breastfeeding in the United States: a pediatric cost analysis. Pediatrics. 2010;125(5):e1048-56. 
24. Zhang J, Yu KF. What's the relative risk? A method of correcting the odds ratio in cohort studies of common outcomes. JAMA 1998;280(19):1690-1.

25. NHS Digital: Hospital Episode Statistics for England. Admitted Patient Care statistics, 2017-18. NHS Digital; 2018. https://digital.nhs.uk/dataand-information/data-tools-and-services/data-services/hospital-episo de-statistics. Accessed 19 November 2019.

26. Office for National Statistics: Population Estimates for UK, England and Wales, Scotland and Northern Ireland: Mid-2017. Office for National Statistics; 2018. https://www.ons.gov.uk/peoplepopulationandcommun ity/populationandmigration/populationestimates/bulletins/annualmidy earpopulationestimates/mid2017. Accessed 18 November 2019.

27. Office for National Statistics: Infant mortality (birth cohort) tables, England and Wales 2016. Office for National Statistics; 2019. https://www. ons.gov.uk/peoplepopulationandcommunity/birthsdeathsandmarriages/ deaths/datasets/infantmortalitybirthcohorttablesinenglandandwales. Accessed 19 Nov 2019.

28. Office for National Statistics: National Life Tables, England, 2015-2017. Office for National Statistics; 2018. https://www.ons.gov.uk/releases/natio nallifetablesuk2015to2017. Accessed 19 November 2019

29. Cancer Resesarch UK: Breast Cancer (C50): average number of new cases per year and age-specific incidence rates per 100,000 population, females, UK, 2014-2016. Cancer Research UK; 2019. https://www.cance rresearchuk.org/health-professional/cancer-statistics/statistics-by-cancer-type/breast-cancer/incidence-invasive\#heading-One. Accessed 19 November 2019.

30. Office for National Statistics: Childbearing for women born in different years, 1920-2002. Office for National Statistics; 2018. https://www.ons. gov.uk/peoplepopulationandcommunity/birthsdeathsandmarriages/ conceptionandfertilityrates/datasets/childbearingforwomenbornindiff erentyearsreferencetable. Accessed 19 November 2019.

31. Lambe M, Hsieh CC, Chan HW, Ekbom A, Trichopoulos D, Adami HO. Parity, age at first and last birth, and risk of breast cancer: a population-based study in Sweden. Breast Cancer Res Treat. 1996;38(3):305-11.

32. Cancer Resesarch UK: Breast Cancer (C50): average number of deaths per year and age-specific mortality rates per 100,000 females, UK, 2014-2016. Cancer Resesarch UK; 2019. Available from: https://www.cancerrese archuk.org/health-professional/cancer-statistics/statistics-by-cancertype/breast-cancer/mortality. Accessed 19 November 2019.

33. Cancer Resesarch UK: Breast Cancer (C50): 2010-2011 Net survival up to ten years after diagnosis, adults (aged 15-99), England and Wales. Cancer Resesarch UK; 2019. https://www.cancerresearchuk.org/sites/default/ files/cstream-node/surv_curve_breast.pdf. Accessed 19 November 2019.

34. Kind P, Hardnab G, Macran S. UK population norms for EQ-5D. Discussion paper 172. York: Centre for Health Economics, The University of York; 1999.

35. Peasgood T, Ward SE, Brazier J. Health-state utility values in breast cancer. Expert Rev Pharmacoecon Outcomes Res. 2010;10(5):553-66.

36. Mariotto AB, Etzioni R, Hurlbert M, Penberthy L, Mayer M. Estimation of the number of women living with metastatic breast Cancer in the United States. Cancer Epidemiol Biomark Prev. 2017;26(6):809-15.

37. Cancer Resesarch UK: Breast Cancer (C50): breast Cancer incidence by stage at diagnosis. Cancer Resesarch UK; 2014. https://www.cancerrese archuk.org/health-professional/cancer-statistics/statistics-by-cancertype/breast-cancer/incidence-invasive\#heading-Three. Accessed 19 November 2019.

38. NHS Improvement: National Schedule of reference costs. Year 2017-18 NHS trusts and NHS foundation trusts. NHS Improvement; 2018. https:// improvement.nhs.uk/resources/reference-costs/. Accessed 19 November 2019.

39. Peres LC. Post-mortem examination in the United Kingdom: present and future. Autops Case Rep. 2017;7(2):1-3.

40. Laudicella M, Walsh B, Burns E, Smith PC. Cost of care for cancer patients in England: evidence from population-based patient-level data. $\mathrm{Br} \mathrm{J}$ Cancer. 2016;114(11):1286-92.

41. National Institute for Health and Care Excellence: Developing NICE guidelines: the manual (last updated October 2020). NICE; 2014. https://WWW. nice.org.uk/process/pmg20. Accessed 19 March 2021.

42. Briggs A, Sculpher M, Claxton K. Decision modelling for health economic evaluation. New York, NY: Oxford University Press; 2006
43. National Institute for Health and Care Excellence: Guide to the Methods of Technology Appraisal 2013 (PMG 9). NICE; 2013 https://www.nice.org. uk/process/pmg9. Accessed 19 November 2019.

44. Higgins JPT, Thomas, J. Cochrane handbook for systematic reviews of interventions, version 6. The Cochrane Collaboration; 2019. https://train ing.cochrane.org/handbook/current Accessed 18 Mar 2020.

45. John EM, Hines LM, Phipps Al, Koo J, Longacre TA, Ingles SA, et al. Reproductive history, breast-feeding and risk of triple negative breast cancer: the breast Cancer etiology in minorities (BEM) study. Int J Cancer. 2018;142(11):2273-85.

46. Ma H, Ursin G, Xu X, Lee E, Togawa K, Duan L, et al. Reproductive factors and the risk of triple-negative breast cancer in white women and AfricanAmerican women: a pooled analysis. Breast Cancer Res. 2017;19(1):6.

47. Narayanan I, Prakash K, Gujral W. The value of human milk in the prevention of infection in the high-risk low-birth-weight infant. J Pediatr. 1981;99(3):496-8.

48. Lucas A, Cole TJ. Breast milk and neonatal necrotising enterocolitis. Lancet. 1990;336(8730):1519-23.

49. Lucas A, Morley R, Cole TJ, Lister G, Leeson-Payne C. Breast milk and subsequent intelligence quotient in children born preterm. Lancet. 1992;339(8788):261-4.

50. Bier JA, Oliver T, Ferguson AE, Vohr BR. Human milk improves cognitive and motor development of premature infants during infancy. J Hum Lact. 2002:18(4):361-7.

51. Severens JL, Milne RJ. Discounting health outcomes in economic evaluation: the ongoing debate. Value Health. 2004;7(4):397-401.

52. Bonneux $L$, Birnie $E$. The discount rate in the economic evaluation of prevention: a thought experiment. J Epidemiol Community Health. 2001;55(2):123-5.

53. Attema AE, Brouwer WBF, Claxton K. Discounting in economic evaluations. Pharmacoeconomics. 2018:36(7):745-58.

54. Haacker M, Hallett TB, Atun R. On discount rates for economic evaluations in global health. Health Policy Plan. 2020;35(1):107-14.

55. Bartick MC, Schwarz EB, Green BD, Jegier BJ, Reinhold AG, Colaizy TT, Bogen DL, Schaefer AJ, Stuebe AM. Suboptimal breastfeeding in the United States: Maternal and pediatric health outcomes and costs. Matern Child Nutr. 2017;13(1):e12366.

56. Büchner FL, Hoekstra J, van Rossum CTM. Health gain and economic evaluation of breastfeeding policies: model simulation. Bilthoven, Netherlands: RIVM; 2007.

57. Colchero MA, Contreras-Loya D, Lopez-Gatell H, Gonzalez de Cosio T. The costs of inadequate breastfeeding of infants in Mexico. Am J Clin Nutr. 2015;101(3):579-86.

58. Ma P, Brewer-Asling M, Magnus JH. A case study on the economic impact of optimal breastfeeding. Matern Child Health J. 2013;17(1):9-13.

59. Rollins NC, Bhandari N, Hajeebhoy N, Horton S, Lutter CK, Martines JC, et al. Lancet breastfeeding series $\mathrm{G}$ : why invest, and what it will take to improve breastfeeding practices? Lancet. 2016;387(10017):491-504

60. Walters DD, Phan LTH, Mathisen R. The cost of not breastfeeding: global results from a new tool. Health Policy Plan. 2019;34(6):407-17.

61. World Health Organization: international code of Marketing of Breast-Milk Substitutes. Geneva:WHO; 1981. https://apps.who.int/iris/handle/10665/ 40382. Accessed 5 Jan 2022.

\section{Publisher's Note}

Springer Nature remains neutral with regard to jurisdictional claims in published maps and institutional affiliations. 